\title{
Strain Localization and Failure in Irradiated Zircaloy with Crystal Plasticity
}

\author{
T.O. Erinosho and F.P.E. Dunne \\ Department of Materials, Imperial College London, SW7 2AZ, United Kingdom
}

\begin{abstract}
This paper presents a micromechanical and mechanistic study of irradiation-induced crystallographic softening known to accelerate failure in irradiated zircaloys typically used as cladding material in pressure water nuclear reactors. The irradiation is known to lead to an increase in yield strength, and reduced ductility is anticipated to result from the progressive reduction in slip system strength. Extensive studies using transmission electron microscopy (TEM) show the formation of $\langle\mathrm{a}\rangle$ type dislocation channels in irradiated zircaloys anticipated to affect basal and prismatic systems. A crystal plasticity approach is established to incorporate basal and prismatic crystallographic softening, both of which are shown to be required in order to capture independent experimental observations for irradiated zircaloy.

Representative irradiated zircaloy textures subjected to cyclic loading regimes were modelled to provide an understanding of the failure processes during in-service conditions. Under both strain and stress-controlled cyclic loading, irradiation softening led to the development of strain localization, and the formation of slip banding and its coalescence. This was found to lead to localized ratcheting and macroscale softening, and in strain-controlled loading, ultimately to plastic shakedown. Stress-controlled cyclic loading, however, especially with non-zero mean applied stress, led to pronounced local and macroscale ratcheting, influenced profoundly by the irradiation softening, and hence finally to ductile failure. It was also observed that local strain hardening due to GND development was small compared to irradiation-induced softening processes, supporting the notion that slip system softening dominates shear band formation.
\end{abstract}

Keywords: Neutron irradiation, strain localization, slip bands, crystal plasticity, cyclic loading, zircaloy. 


\section{Introduction}

This paper addresses the mechanisms affecting the structural integrity of Zircaloy subjected to irradiation during in-service nuclear application. The consequences of neutron irradiationinduced slip system softening in reactor cladding material (often zircaloys) are investigated to understand the deformation mechanisms and the nature of irradiation-accelerated strain localization. Some work to explain the micromechanical mechanisms of deformation resulting from irradiation has been undertaken using computational plasticity and supported by experimental observation and has been reported in the literature. So far, the numerical studies used employ self-consistent models [1,2] which are known to be useful for addressing macroscopic material response based on average ensembles of material orientations, but equally are less good in understanding local, grain-by-grain, slip system based-deformation. This is because of the simplifications involved in abandoning either equilibrium or compatibility (or both) requirements, and because they do not represent the details of grain morphology and interaction fully. However, the crystal plasticity framework adopted here overcomes these limitations and offers the possibility of a better understanding of slip-based deformations, and particularly strain localization, in $\mathrm{Zr}$ alloys resulting from slip system softening known to result from neutron irradiation.

Material reliability and safety are crucial to industrial practices and hence, it is no coincidence that extensive interest exists in understanding deformation mechanisms in hexagonal close packed (HCP) metals such as Zircaloys, given their range of applications in critical and highly sensitive environments. Zircaloys are important materials used for cladding in nuclear reactors and are subjected to high levels of irradiation, or fluence. Experiments have shown that changes in material crystallographic-level structure arise due to fluence thereby raising safety concerns [3-5].

The effect of fluence on zircaloys can be macroscopic and microscopic. Macroscopically, fluence leads to an increase in yield strength of the material [1,4] and lowering in the ductility, i.e. strain to failure, shown experimentally in Fig. 1. Microscopically, it affects the differing classes of slip systems found in zircaloys [1]. Zircaloys have four classes of slip systems illustrated in Fig. 2 which are basal, prismatic, <a> pyramidal and $\langle\mathrm{c}+\mathrm{a}>$ pyramidal types with differing critical resolved shear stresses (CRSS) on each system detailed in Table 1 for a particular zircaloy of interest. The CRSS determines the ease of achieving slip on that particular slip system and in Zircaloys, the basal and prismatic systems generally have lower CRSS compared to the pyramidal systems [2]. The CRSS for prismatic systems is usually lower than that for basal systems [4]. Experiments on Zircaloys, however, strongly suggest that the CRSS on these slip systems change by varying degrees with fluence and the increase can be estimated $[1,2]$. There are also indications that the CRSS for prismatic systems after irradiation is higher than that for basal systems i.e. a strength reversal compared to the unirradiated material.

Transmission electron microscopy (TEM) analysis shows that pronounced slip localization along dislocation channels, illustrated in Fig. 3, is observed in irradiated zircaloys compared to unirradiated samples [3-6]. This has been attributed to the efficient clearing of dislocation 
channels leading to increased slip activity along these channels [4]. A further consequence of such channels is the increased susceptibility of the irradiated material to stress corrosion cracking (SCC), especially where the channels intersect with surfaces [7].

Furthermore, irradiation-induced softening on active slip systems promotes accelerated localization and consequently, lower strain to failure [8]. This phenomenon is suggested to affect basal slip systems, but also prismatic systems to a lesser extent, but it remains unclear at what rate the softening on each system type occurs [2,9]. Slip system softening however, manifesting as a decrease in CRSS, is thought to be bounded [2] and does not decrease beyond the CRSS of the unirradiated material.

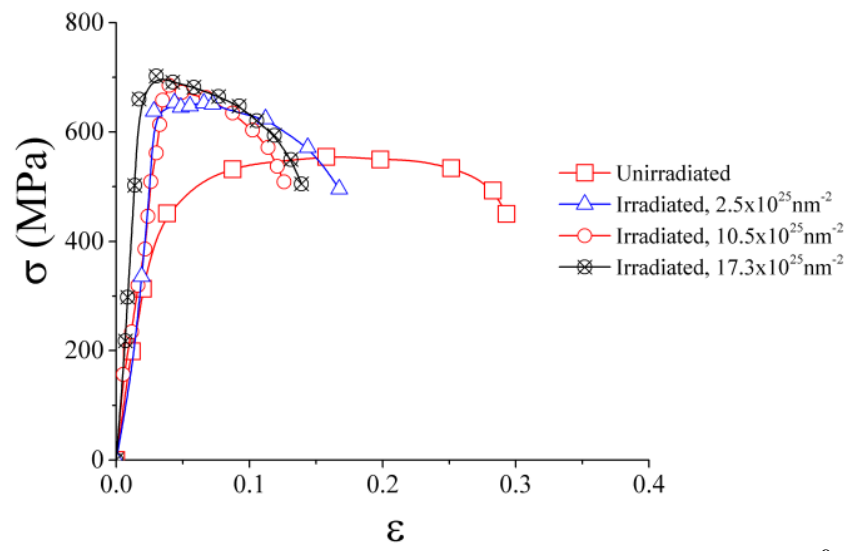

Fig. 1. Typical $\sigma-\varepsilon$ curves of the irradiated/unirradiated Zircaloy at a test temperature of $23^{\circ} \mathrm{C}$ reproduced from Wei et al. [4].
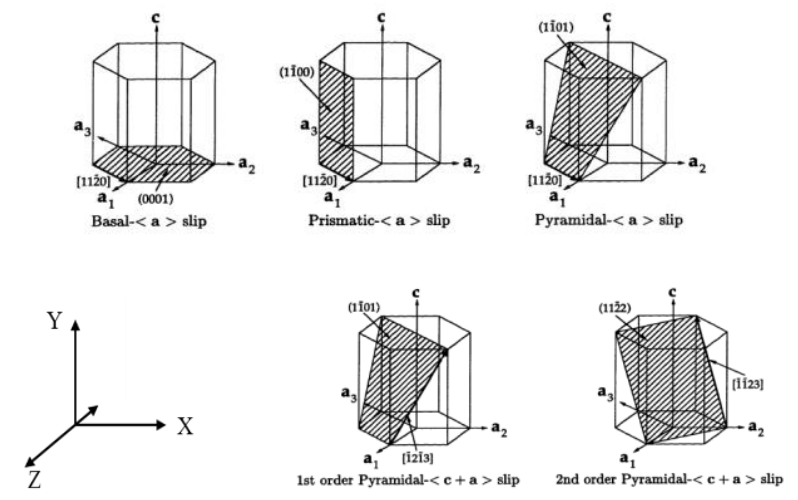

Fig. 2. The range of HCP slip systems incorporated into the crystal plasticity calculations each having CRSS values specified in Table 1.

Table 1: Micromechanical properties of zircaloy used in this study. The increases in CRSS for the differing slip systems are obtained from Onimus et al. [2] and Francillette et al. [10] for a fluence level of $\mathbf{5} \times \mathbf{1 0}^{\mathbf{2 5}} \mathbf{n m}^{-\mathbf{2}}$.

\begin{tabular}{|c|c|c||c||c|}
\hline & Basal & Prismatic & <a>Pyramidal & <c+a>Pyramidal \\
\hline \hline$\left(\tau_{0}^{\alpha}\right)^{\text {un-irra }}(\mathrm{MPa})$ & 162.5 & 125 & 500 & 500 \\
\hline$\Delta \tau(\mathrm{MPa})$ due to irradiation & 109 & 204 & 189 & 318 \\
\hline$\left(\tau_{0}^{\alpha}\right)^{\text {irra }}(\mathrm{MPa})$ & 271.5 & 329 & 689 & 818 \\
\hline
\end{tabular}




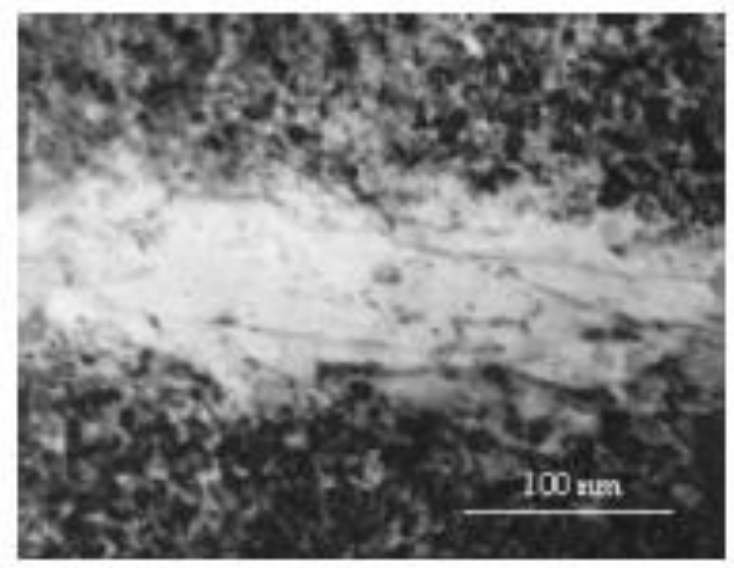

Fig. 3. Example of dislocation channel formed in irradiated Zircaloy after tensile testing reproduced from Wei et al. [4]

This paper attempts to understand two key issues. It addresses (i) the onset and rate of softening on individual slip systems, and perhaps more importantly, (ii) the strain localization effects resulting from irradiation-induced softening. The former involves the development of a slip-system dependent softening model in order to reproduce the experimentally observed mechanical response for representative irradiated zircaloy textures. The latter entails the nucleation and growth of strain localization and slip bands in the irradiated model material, utilizing the softening model developed, and their consequences for deformation behaviour under representative in-service loading regimes.

An assessment of a number of possible slip system weakening mechanisms contributing to softening responses is presented in $\S 2$, together with a brief summary of the crystal plasticity modelling adopted. Sections $\S 3$ and $\S 4$ address the effects of neutron irradiation-induced softening in single and polycrystal zircaloys with differing textures, and this is followed by an assessment of polycrystal behaviour for a commonly employed texture under representative in-service cyclic loading. Finally, conclusions are presented in $§ 5$.

(a)

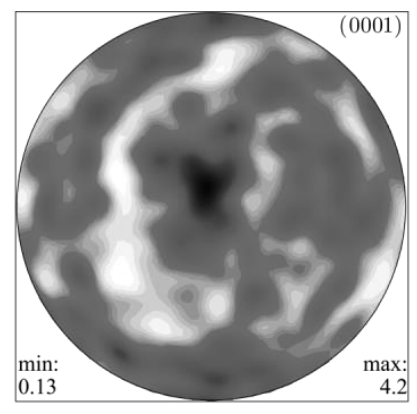

(b)

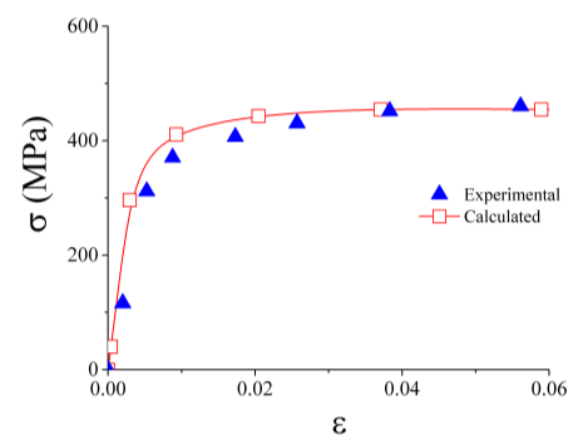

Fig. 4. Experimental $\sigma-\varepsilon$ data used to calibrate the crystal plasticity model. A representative zircaloy texture shown in (a) was subjected to uniaxial straining and the CRSSs for the HCP slip systems were calibrated to match the experimentally obtained stress-strain response shown in (b) for Zircaloy-2 presented by Wen et al.[11]. 


\section{Micromechanical mechanisms affecting slip-system strength during zircaloy irradiation}

Mechanical tests on irradiated samples show an initial significant increase in yield strength reflecting increases in slip system strengths. The magnitudes of the increases have been investigated such that they can be estimated according to [2] using

$\Delta \tau^{\alpha}=\chi^{\alpha} \mu b^{\alpha} \sqrt{N d}$

where $\Delta \tau^{\alpha}$ is the change in the CRSS on a particular slip system $\alpha$ and $b$ is the Burger's vector on slip system $\alpha$ such that $b^{\text {basal }}=b^{\text {pris }}=b^{<\mathrm{a}>\text { pyra }}=0.295 \mathrm{~nm}$ and $b^{<\mathrm{c}+\mathrm{a}>\text { pyra }}=$ $0.552 \mathrm{~nm}$. $N=5 \times 10^{22} \mathrm{~m}^{-3}$ is the loop density and $d=10 \mathrm{~nm}$ the loop diameter which saturates for fluences above $5 \times 10^{25} \mathrm{~nm}^{-2}$ [12]. $\mu=33000 \mathrm{MPa}$ is the shear modulus and $\chi$ is a parameter which characterizes the ease of slip on a particular slip system $\alpha$ such that $\chi^{\text {basal }}=0.5, \chi^{\text {pris }}=0.94, \chi^{<\mathrm{a}>\text { pyra }}=0.87$ and $\chi^{<\mathrm{c}+\mathrm{a}>\text { pyram }}=0.78[13,14]$. Table 1 provides a summary of the material properties adopted in this study. Note that in the unirradiated state, prismatic slip has the lowest CRSS but basal slip requires a lower resolved shear to cause slip in the irradiated material. A subsequent and further manifestation of irradiation is progressive slip system softening attributed to efficient clearing of dislocations along dislocation channels [2]. That is, $\tau_{0}$ on a particular slip system $\alpha$ reduces progressively due to irradiation and reduces from the irradiated level back down towards its unirradiated value detailed in Table 1.

The two manifestations detailed above have been studied using dislocation dynamics by Drouet et al. [15]. The advantage of dislocation dynamics is the ability to evaluate in detail dislocation glide and interactions under an applied stress. Their study presented a systematic evaluation of the relative effects of edge and screw type dislocations gliding on basal and prismatic planes. A square loop with two components of the loop on basal and two on prismatic planes was introduced ahead of a Frank Read source in the dislocation dynamics simulation box which was then subjected to pure shear in the gliding plane of dislocations. $10 \mathrm{~nm}$ side length of the square loop was chosen based on TEM analysis on typical lengths of dislocation loops in irradiated zirconium [16, 17]. Further, in order to fully represent microstructure based parameters in irradiated zirconium, Drouet et al. [15] chose the loop density based on the TEM studies. The loop density is defined as the distance between the centers of each introduced loop within the simulation box.

Drouet et al. [15] showed that dislocation pinning was more pronounced in the prismatic compared to the basal system accounting for the higher CRSS reported for the prismatic compared to the basal slip systems in irradiated zirconium. Note that in the unirradiated material, the prismatic system has a lower CRSS compared to the basal system. Further, their analysis showed dislocation glide occurs on both basal and prismatic planes but whilst full clearing of dislocation channels occurred on basal systems, only partial clearing of dislocations was seen on prismatic systems. This was attributed to favorable interaction of dislocations (edge or screw) on basal planes leading to the formation of superjogs which have the ability to clear the dislocation channel. Nevertheless, Drouet et al. [15] indicate strongly 
that dislocation channeling occurs on both basal and prismatic systems and that both systems potentially contribute to the accelerated irradiation induced localization evident in the macroscopic mechanical response of zircaloys.

Moreover, dislocation channeling on both basal and prismatic systems has been observed experimentally by Onimus et al. [17] using TEM on irradiated zirconium. The grains in all samples considered were first mapped using conventional diffraction pattern indexing in order to obtain the local orientation and stereographic projection. Subsequently, traces of channels within each grain were then analyzed at high magnification onto the stereographic projection. Using this technique, the number of channels and mean channel width were recorded. They reported that basal channels were frequently observed and whilst prismatic channels are more difficult to activate, they are also present. In addition, the mean width of the channels was observed to be $40 \mathrm{~nm}$ and the maximum width being $50 \mathrm{~nm}$. This study therefore provides further evidence of prismatic chanelling in irradiated zircaloys.

Hence, in order to attempt to understand the softening occuring in irradiated zircaloy, a range of hypotheses are tested against the experimental observations presented by Wei et al. [4]. The hypotheses have been developed on the basis that softening primarily affects the basal systems, as widely reported in the literature, but in addition the effects of secondary softening mechanisms such as that occurring on prismatic systems (as opposed to pyramidal systems) are also then considered $[2,3,5,6]$ and indeed are then subsequently argued to be essential in order to capture experimentally observed strain localization and failure.

It is proposed to consider that irradiation-induced slip system softening affects:

i. only basal slip and a critical value of basal slip $\left(\gamma^{\text {crit }}>0\right)$ exists such that once achieved, slip system softening progressively develops (Case I).

ii. only basal slip and that softening initiates at the first onset of $\operatorname{sip} \gamma^{\text {crit }}=0$ on that slip system (Case II).

iii. both basal and prismatic slip at different rates and is initiated at the first onset of slip $\gamma^{\text {crit }}=0$ on these slip systems (Case III).

iv. both basal and prismatic slip at the same rate and a critical value of $\operatorname{sip}\left(\gamma^{\text {crit }}>0\right)$ exists (Case IV).

Consider a grain $i$ which is discretized into many elements within our finite element (FE) model. Softening is introduced at every integration point $j$ within grain $i$ which satisfies the softening criterion detailed above such that

$\tau_{j_{\text {basal }}^{t+\Delta t}}^{t+\tau_{\text {basal }}^{\text {irra }}}-f(\xi)_{j_{\text {basal }}} \Delta \tau_{\text {basal }}$

and for prismatic systems,

$\tau_{j_{\text {pris }}}^{t+\Delta t}=\tau_{\text {pris }}^{\text {irra }}-f(\xi)_{j_{\text {pris }}} \Delta \tau_{\text {pris }}$

where $\tau_{\text {basal }}^{\text {irra }}$ and $\tau_{\text {pris }}^{\text {irra }}$ are the initial CRSS values on the basal and prismatic slip systems respectively in element $j$ after irradiation hardening, but prior to any subsequent basal or 
prismatic softening, and $\Delta \tau_{\text {basal }}$ and $\Delta \tau_{\text {pris }}$ represent the decreases in CRSS from the hardened irradiated state to the fully softened state, which is taken here to be the same as the original unirradiated values, as detailed in Table 1 . That is, any softening that occurs cannot lead to a CRSS less than the original unirradiated level [2].

A linear softening parameter, $f(\xi)$ has been introduced for the basal and prismatic slip system CRSSs respectively such that its parameters can be calibrated to investigate the range of hypotheses presented above, and these are detailed as follows.

Case I: Irradiation induced softening affects basal slip systems only and is initiated at a critical value of slip such that

$$
f(\xi)_{\text {basal }}= \begin{cases}0 & \gamma^{\text {basal }} \leq \gamma^{\text {crit }} \\ \frac{\gamma-\gamma^{\text {crit }}}{\gamma_{\text {basal }}^{\text {sat }}-\gamma^{\text {crit }}} & \gamma^{\text {basal }}>\gamma^{\text {crit }}\end{cases}
$$

and

$$
f(\xi)_{\text {pris }}=0 \text {. }
$$

Case II: Irradiation induced softening affects only basal slip systems and initiates at the onset of slip such that

$$
f(\xi)_{\text {basal }}= \begin{cases}0 & \gamma^{\text {basal }} \leq 0 \\ \frac{\gamma-\gamma^{\text {crit }}}{\gamma_{\text {basal }}^{\text {sat }}-\gamma^{\text {crit }}} & \gamma^{\text {basal }}>0\end{cases}
$$

and

$$
f(\xi)_{\text {pris }}=0 \text {. }
$$

Case III: Irradiation induced softening affects basal and prismatic slip systems at different rates and is initiated at the onset of slip on each slip system such that

$$
f(\xi)_{\text {basal }}= \begin{cases}0 & \gamma^{\text {basal }} \leq 0 \\ \frac{\gamma-\gamma^{\text {crit }}}{\gamma_{\text {basal }}^{\text {sat }}-\gamma^{\text {crit }}} & \gamma^{\text {basal }}>0\end{cases}
$$

and

$$
f(\xi)_{\text {pris }}= \begin{cases}0 & \gamma^{\text {pris }} \leq 0 \\ \frac{\gamma-\gamma^{\text {crit }}}{\gamma_{\text {pris }}^{\text {sat }}-\gamma^{\text {crit }}} & \gamma^{\text {pris }}>0\end{cases}
$$

Case IV: Irradiation induced softening affects both basal and prismatic slip systems at the same rate and is initiated at a critical level of slip such that 


$$
f(\xi)_{\text {basal }}= \begin{cases}0 & \gamma^{\text {basal }} \leq \gamma^{\text {crit }} \\ \frac{\gamma-\gamma^{\text {crit }}}{\gamma_{\text {basal }}^{\text {sat }}-\gamma^{\text {crit }}} & \gamma^{\text {basal }}>\gamma^{\text {crit }}\end{cases}
$$

and

$$
f(\xi)_{\text {pris }}= \begin{cases}0 & \gamma^{\text {pris }} \leq \gamma^{\text {crit }} \\ \frac{\gamma-\gamma^{\text {crit }}}{\gamma_{\text {pris }}^{\text {sat }}-\gamma^{\text {crit }}} & \gamma^{\text {pris }}>\gamma^{\text {crit }}\end{cases}
$$

A saturation value of $\operatorname{slip}\left(\gamma^{\text {sat }}\right)$ is prescribed (and detailed later) to control the rate of softening and for all analyses considered, the pyramidal slip systems are unaffected i.e. neither softening nor hardening is implemented. Note that $0 \leq f(\xi) \leq 1$ has been prescribed to ensure that a fully softened slip system cannot have a lower strength than in its unirradiated state.

The studies considered in this work investigate channel softening on both basal and prismatic slip systems for which both the onset of softening and the rate of softening are both explicitly examined, since the currently available experimental evidence supports the existence of softening on both slip system sets, but does not at present allow for the quantitative measurement of the form of the softening. Hence, only linear slip system softening with accumulated slip is considered, but extension of the model to include other forms of softening is trivial.

\subsection{Crystal plasticity model}

Slip has been demonstrated as the dominant mechanism for deformation in irradiated zircaloys at room temperature. Although, deformation by twinning is frequently observed at low temperatures in irradiated zircaloys, early studies by Onchi [18] report that neutron irradiation inhibits twinning at room temperature. Two reasons have been potentially attributed for this: (1) direct suppression of twin growth by irradiation induced defects and (2) relaxation of twinning stress due to irradiation induced dislocation structures. The former is related to the significant development of dislocation loops which act as inhibitors to twin growth whilst the latter suggests a high twin stress required to achieve growth.

The slip based crystal plasticity framework adopted is standard and based on the kinematic decomposition of the deformation gradient $\boldsymbol{F}$ into elastic $\left(\boldsymbol{F}^{\mathrm{e}}\right)$ and plastic $\left(\boldsymbol{F}^{\mathrm{p}}\right)$ tensors as described by Lee [19] such that

$\boldsymbol{F}=\boldsymbol{F}^{\mathrm{e}} \boldsymbol{F}^{\mathrm{p}}$

Deformation is accommodated on any given HCP slip system (basal, prismatic, <a> pyramidal and $<\mathrm{c}+\mathrm{a}>$ pyramidal) and contributes to the overall plastic deformation given by

$\boldsymbol{F}^{\mathrm{p}}=\boldsymbol{I}+\sum \gamma^{\alpha}\left(\boldsymbol{s}^{\alpha} \otimes \boldsymbol{n}^{\alpha}\right)$ 
The plastic part of the velocity gradient is decomposed into symmetric $\boldsymbol{D}^{\boldsymbol{p}}$ and antisymmetric $\boldsymbol{\Omega}^{p}$ parts

$\boldsymbol{L}^{\mathrm{p}}=\sum \dot{\gamma}^{\alpha}\left(\boldsymbol{s}^{\alpha} \otimes \boldsymbol{n}^{\alpha}\right)=\boldsymbol{D}^{\mathrm{p}}+\boldsymbol{\Omega}^{\mathrm{p}}$.

The evolution of texture is determined from the antisymmetric part of the elastic velocity gradient, $\boldsymbol{\Omega}^{\mathbf{e}}$, given by

$\boldsymbol{\Omega}^{\mathrm{e}}=\operatorname{asym}(\boldsymbol{L})-\operatorname{asym}\left(\left(\sum \dot{\gamma}^{\alpha} \boldsymbol{s}^{\alpha} \otimes \boldsymbol{n}^{\alpha}\right)\right.$

and the slip ( $\left.\boldsymbol{s}^{\alpha}\right)$ and normal $\left(\boldsymbol{n}^{\alpha}\right)$ directions are updated using $\boldsymbol{s}^{\alpha^{*}}=\boldsymbol{\Omega}^{\mathrm{e}} \boldsymbol{s}^{\alpha}$ and $\boldsymbol{n}^{\alpha^{*}}=$ $\boldsymbol{n}^{\alpha} \boldsymbol{\Omega}^{\mathrm{e}-1}$ respectively.

The slip rule adopted is simply assumed to be a power law relationship between slip rate and resolved shear stress for a given slip system and is given by

$\dot{\gamma}^{\alpha}=\dot{\gamma}_{0}\left|\frac{\tau^{\alpha}}{\tau_{C}^{\alpha}}\right|^{n} \operatorname{sgn}\left(\dot{\gamma}^{\alpha}\right) \quad$ and $\tau_{c}^{\alpha}=\tau_{0}^{\alpha}+G b \sqrt{\rho_{G}^{\alpha}}$

where the slip rate $\left(\dot{\gamma}^{\alpha}\right)$ evolves on individual slip systems $(\alpha)$ and depends on the reference strain rate, $\dot{\gamma}_{0}^{\alpha}$, resolved shear stress, $\tau^{\alpha}$, and CRSS on a particular slip system, $\tau_{c}^{\alpha}$. The CRSS may be increased by virtue of the development of GND density as shown in Eq. (8) in which Taylor hardening is assumed and $G$ is shear modulus and $b$ the corresponding Burger's vector magnitude. Full details of this model may be found in [20, 21]. The CRSSs $\tau_{0}^{\alpha}$ on each slip system, corresponding to the unirradiated and irradiated material states respectively are detailed in Table 1 which were calibrated against the experimental stress-strain data shown in Fig. 4.

For the unirradiated case, a representative zircaloy texture shown in Fig. 4a was subjected to uniaxial straining and the CRSSs on each slip system were calibrated to match the experimentally obtained stress-strain response shown in Fig. 4b. A fixed ratio, presented by Francillette et al. [22], is adopted between the relative slip system strengths during calibration such that $\tau_{0}^{\text {basal }}=1.3 \tau_{0}^{\text {pris }}$ and $\tau_{0}^{\text {pyra }}=4 \tau_{0}^{\text {pris }}$. A detailed discussion is presented in Appendix A to compare the range of CRSS values for individual slip systems reported in the literature for Zircaloys, together with a polycrystal model assessment of the consequent macro-level uniaxial stress-strain response which results.

The CRSSs in the irradiated material were obtained by adopting Eq. (1) to calculate the increase in CRSS on individual slip systems given in Table 1 such that

$\left(\tau_{0}^{\alpha}\right)^{\text {irra }}=\left(\tau_{0}^{\alpha}\right)^{\text {un-irra }}+\Delta \tau^{\alpha}$

where $\left(\tau_{0}^{\alpha}\right)^{\text {irra }}$ is the CRSS on slip system $\alpha$ in the irradiated state and $\left(\tau_{0}^{\alpha}\right)^{\text {un-irra }}$ is the unirradiated CRSS.

Further, the development and evolution of geometrically necessary dislocations (GNDs) is potentially important especially in the context of strain localization. GNDs are known to contribute localized strain hardening at small length scales typically less than $10 \mu \mathrm{m}$. It is 
firstly noted that forest hardening is not explicitly accounted for in this model, but the effects of GND development are considered to evaluate whether they are significant during irradiation induced slip system softening. The evolution of GNDs is accounted by adopting the methodology presented by Erinosho et al.[23] such that the net Burger's vector $(\boldsymbol{B})$ of all dislocations piercing through an infinitesimal surface, $(S)$, with normal $\boldsymbol{n}$, and enclosed counterclockwise by a circuit, $\Gamma$ is translated to a GND density using stokes theorem. That is,

$\boldsymbol{B}=\oint_{\Gamma} \boldsymbol{F}^{\mathrm{P}} d \boldsymbol{X}=\iint_{S} \operatorname{curl}\left(\boldsymbol{F}^{\mathrm{p}}\right) \boldsymbol{n} d S$.

An equivalent expression for Eq. (10) is given in (11)

$\boldsymbol{B}=\iint_{S}\left(\boldsymbol{\rho}_{G} \cdot \boldsymbol{r}\right) \boldsymbol{b}_{G} d S \equiv \iint_{S}\left(\boldsymbol{b}_{G} \otimes \boldsymbol{\rho}_{G}\right) \boldsymbol{n} d S$

such that the discontinuity vector $\boldsymbol{B}$ may also be obtained from consideration of the net GND density $\boldsymbol{\rho}_{G}$ by finding its component in the direction normal to the plane, $\boldsymbol{n}$ with consideration of the net GND Burger's direction, $\boldsymbol{b}_{G}$. From Eqs. (10) and (11), the density of GNDs can be obtained such that

$\sum_{\alpha=1}^{N} \boldsymbol{b}_{\mathrm{G}}^{\alpha} \otimes \boldsymbol{\rho}_{\mathrm{G}}^{\alpha} \equiv \operatorname{curl}\left(\boldsymbol{F}^{\mathrm{p}}\right)$

where the GND densities are summed over all slip systems $(\alpha)$ of edge and screw type. By accounting for edge and screw type burgers vectors within the HCP formulation, it becomes apparent that Eq. (12) is under-defined and requires a numerically approximate solution. Here, the $L^{1}$ method based on energy minimisation which considers any dislocation as a line through the volume was adopted. The dislocation line energy is then minimized by finding the dislocation configuration with the smallest total line length [24]. The evolution of GND density is calculated in the present study to enable the evaluation of the potential contribution of strain gradient hardening compared to irradiation induced softening.

In the next section, the range of softening hypotheses are assessed for both single crystal and differing polycrystal texture behaviour when subjected to uniaxial straining. Further, representative in-service loading conditions are also considered by assessing a common polycrystal texture used in cladding material under cyclic loading to investigate softening, localization, ratcheting, and failure.

\section{Irradiation-induced slip localization and softening in single and polycrystal zircaloy}

Single crystal zircaloy is examined first, subjected to uniaxial straining, to provide a basic understanding of the progressive formation of localized slip bands and their effect on macroscopic mechanical response. The single crystal ABAQUS (Explicit) C3D8R model shown in Fig. 5a comprises 12,400 linear 8 noded elements with initial crystallographic orientation (C-II) shown in Fig. 5b was subjected to uniaxial straining in the Y-direction illustrated schematically in Fig. 5c. The crystallographic orientation (C-II) was chosen to favour both basal and prismatic slip and the boundary conditions are such that all surfaces are constrained to remain planar. 
Case I (that is, basal softening alone) in the hypotheses stated above is considered first, and Fig. 6 shows a range of accumulated slip field analyses in the single crystal for unirradiated and irradiated cases. The slip field for the unirradiated single crystal subjected to 5 percent uniaxial straining in the Y-direction is shown in Fig. 6a, and that for the corresponding irradiated single crystal without basal softening in Fig. 6b. The softening regime shown in Fig. $6 \mathrm{c}$ is then introduced (in which the critical and saturated slip levels are defined) for the basal slip systems and the progressive effects of basal softening with increasing strain are presented in Figs. 6d-f.

First consider the cases without basal softening i.e. the accumulated slip in the unirradiated single crystal (Fig. 6a) and the irradiated single crystal without basal softening (Fig. 6b). Although not pronounced, a gradual development of slip banding is present in both cases in the Y-Z plane, with higher levels of accumulated strain shown to develop in the unirradiated crystal because of the lower slip strengths reflected in Table 1. With the introduction of the Case I softening regime shown in Fig. $6 \mathrm{c}$, with $\gamma_{\text {basal }}^{\text {crit }}=0.02$, and saturation value $\gamma_{\text {basal }}^{\text {sat }}=$ 0.12 , very significant slip localization and shear banding may be seen to develop in Figs. 6df.

(a)

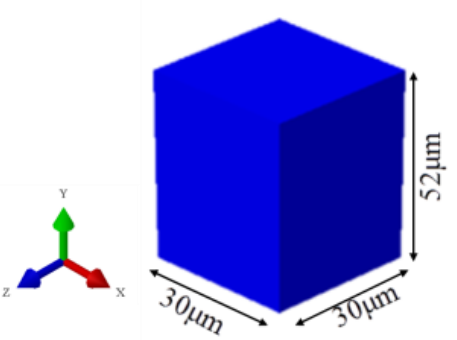

(b)

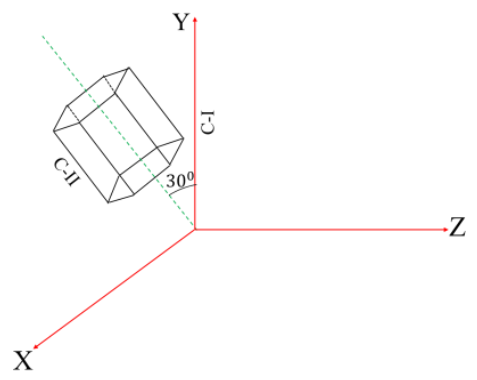

(c)

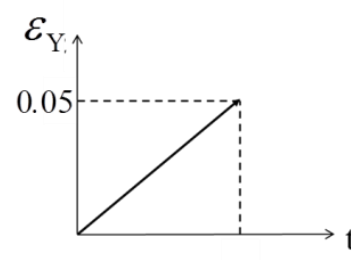

Fig. 5. Single crystal used in the simulations showing the (a) dimensions, (b) initial crystallographic orientation and (c) the loading history

The slip localization is pronounced due to softening on the basal slip systems and this is further emphasized in Fig. 7 which shows the accumulated slip on basal, prismatic and pyramidal systems at 5\% applied strain. It is clear that softening on the basal system results in the significant localization observed in Fig. 7a and less for the prismatic systems in Fig. 7b. Further, it is worth pointing out that the shear stress on the pyramidal slip systems is insufficient to activate slip due to the high CRSS on these systems as seen in Figs. 7c and 7d. The corresponding average stress response of the single crystal with and without softening is shown in Fig. 8, with basal softening and slip localization leading to a substantial stress drop. A systematic study of the relative effects of basal and prismatic softening is carried out for a range of polycrystal textures presented next.

\subsection{Softening in polycrystal zircaloy}

The effects of irradiation-induced softening on zircaloys is next assessed for differing polycrystal textures subjected to uniaxial straining. The response of a contrived single crystal oriented favorably for slip indicated that irradiation-induced softening of basal only systems led to the formation of localization of slip and the development of shear banding. The development and growth of localized slip bands in polycrystal deformation across grains and 
the consequent effects of localization on the macroscopic mechanical response are investigated here. A number of differing initial textures are investigated and incorporated into a representative oligocrystal and subjected to monotonic loading and subsequently, cyclic loading to provide representative in-service responses to irradiation softening.

Wei et al. [4] observed dislocation channels in representative Zircaloy textures used as cladding material in pressure water reactors. They examined the mechanical responses at differing levels of fluence (irradiation) and further, also indicated the consequent lowering in ductility. It was strongly suggested that basal slip accounts mainly for the observed mechanical effect but that other systems are also affected, but to a lesser extent. On the basis of these experimentally observed phenomena, the softening hypotheses presented above are tested in order to establish whether the experimentally observed softening in polycrystals may result solely from basal softening, or indeed whether contributions are also necessary from prismatic softening in order to reproduce the experimentally observed behaviour.

The hypotheses have been outlined in section 3 (Case I to Case IV). However, in this section, focus is placed on Cases II, III and IV and the consequences of these assumptions on resultant deformation are tested next. Investigations are carried out using the oligocrystal model shown in Fig. 9a subjected to the schematic uniaxial loading condition in Fig. 9b. The boundary conditions are such that all negative faces remain planar, the positive surfaces are unconstrained and displacement controlled monotonic loading is applied on the positive-Y surface. The boundary conditions were chosen to impose a uniaxial strain state on the polycrystal, but allowing all positive faces to deform freely, thus representing polycrystal sample free surfaces. However, an additional example analysis is presented in Appendix B in which all of the initial positive and negative orthogonal polycrystal faces are constrained to remain planar in order to provide comparison of the two extreme sets of boundary conditions. Appendix B provides slip fields developed and an assessment of the effects of these boundary conditions. Three representative textures are investigated as shown in Figs. 9c-e. Fig. 9c is a nominally random texture (Texture-A) with a slight preference in the [010] direction while Figs. 9d and 9e have initially preferred orientations. Texture-B and Texture-C shown in Fig. 9 are indicative of extremes; that is, the average c-axis in Texture-B is oriented approximately parallel to the $\mathrm{X}$-direction while in Texture- $\mathrm{C}$, the representative c-axis is perpendicular to the $\mathrm{X}$-direction. Texture- $\mathrm{C}$ is also representative of that employed in nuclear reactor applications, but Texture- $\mathrm{B}$ is also investigated in order to address mechanistic understanding. The textures are incorporated into the oligocrystal (Fig. 9a) with 216 grains comprising $5 \times 5 \times 10$ elements per grain. In passing, it is well known that the accuracy of oligocrystal models in predicting localization using finite element frameworks depends critically on the level of mesh refinement. Hence, a study of mesh refinement levels $(6 \times 6 \times$ 11 and $9 \times 9 \times 15$ elements per grain) was carried out and summarized in Appendix $C$ and shows that adopting this level of refinement $(5 \times 5 \times 10$ elements per grain $)$ is sufficient to capture the onset of slip, localization and failure.

The oligocrystal in the unirradiated state is considered first to act as control and subsequently, softening is introduced on basal slip systems followed by basal and prismatic systems. Fig. 10 
shows the softening regimes adopted for these systems. Note that the softening is introduced at different rates as described in section 3 . 
Un-irradiated $\left(\varepsilon_{Y}=0.05\right)$

(a)
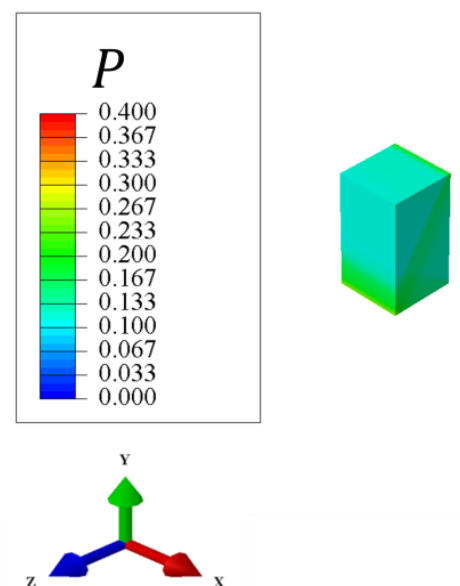

Irradiated with basal softening $\left(\varepsilon_{Y}=0.02\right)$

(d)

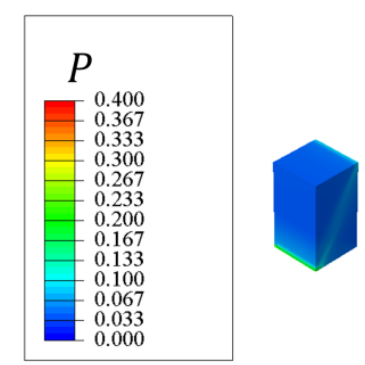

Irradiated without softening $\left(\varepsilon_{Y}=0.05\right)$

(b)
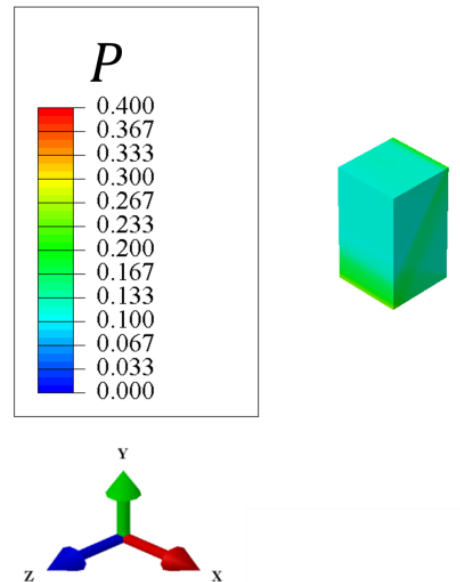

Irradiated with basal softening $\left(\varepsilon_{Y}=0.03\right)$

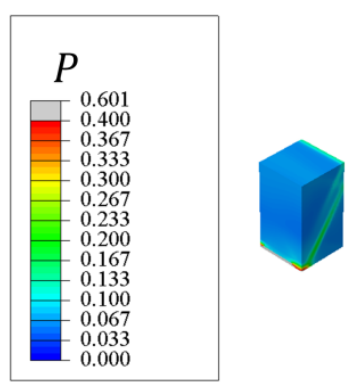

(c)

Basal softening regime

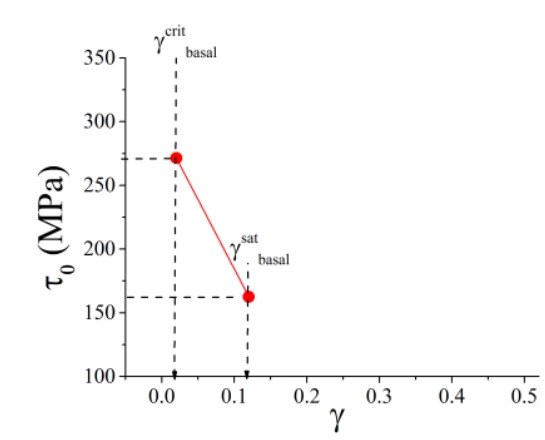

(e)

Fig. 6: Study of irradiation induced localization in single crystal zircaloy shown in Fig. 5a. Examples of the accumulated slip are shown for the (a) unirradiated and (b) irradiated without basal softening. The softening regime shown in (c) is implemented on the basal slip systems and the progressive increase in accumulated slip with strains is shown in (d), (e) and (f). 
(a)
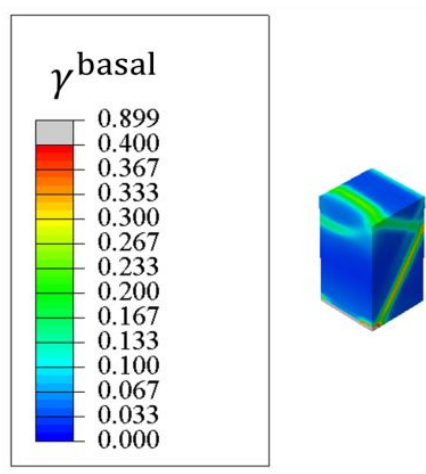

(c)

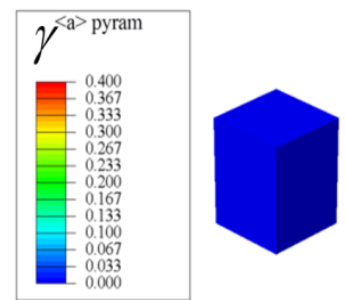

(b)

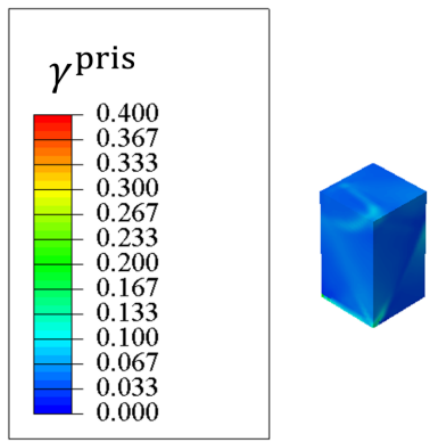

(d)

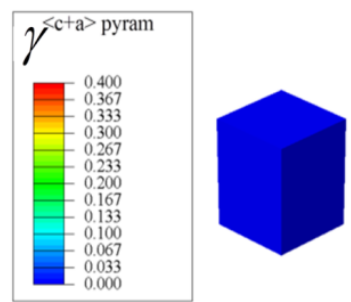

Fig. 7: Study of the accumulated slip on individual slip systems at 5 percent uniaxial straining shown in Fig. $6 f$ showing the basal slip system contributes to the localization observed. Note that the shear stresses on the pyramidal planes are insufficient to activate slip.

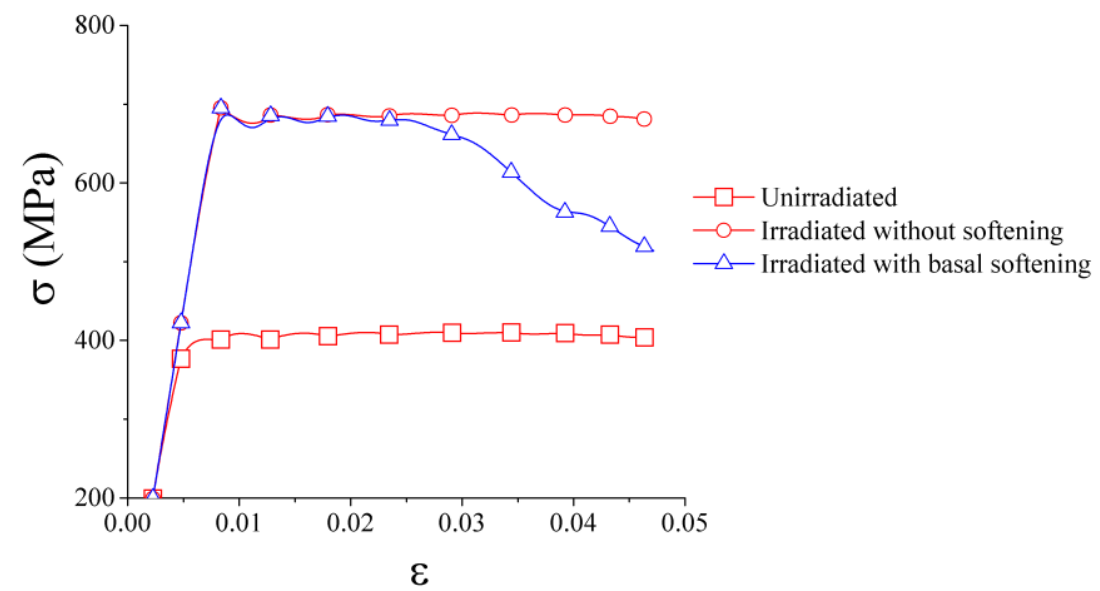

Fig. 8: The macroscopic $\sigma-\varepsilon$ curve for the single crystal zircaloy show in Fig. 5a subjected to 5 percent uniaxial strain. Note the effect of localization typified by a stress drop observed in the irradiated case with basal softening initiated at a critical level of slip on the basal slip system $\left(\gamma_{\text {basal }}^{\text {crit }}=0.02\right)$. 
(a)

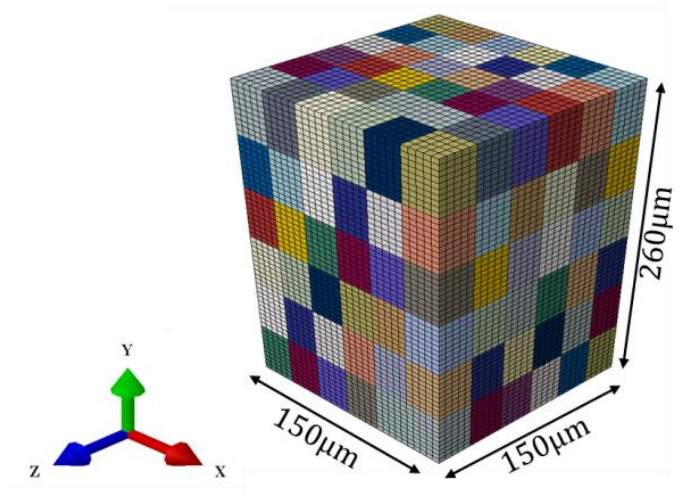

(b)

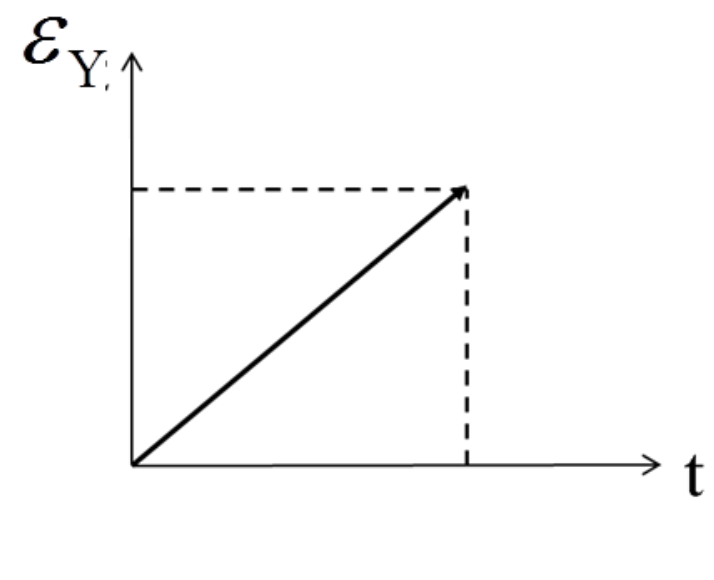

(c)

\section{Texture-A}

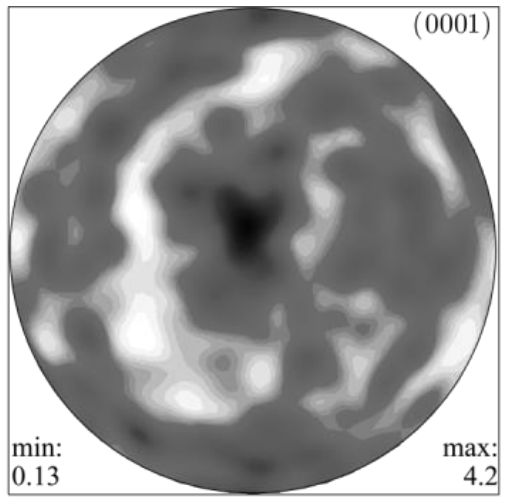

(d)

\section{Texture-B}

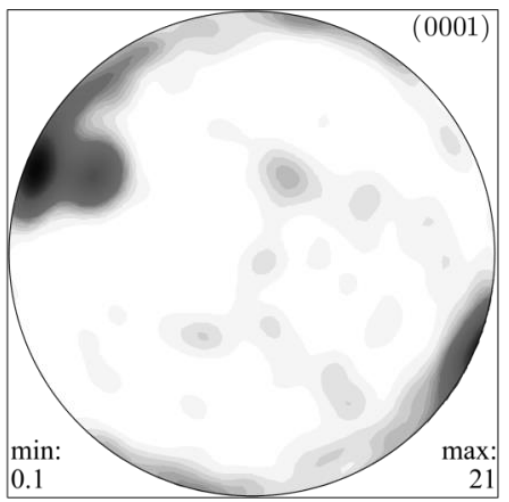

(e)

\section{Texture-C}

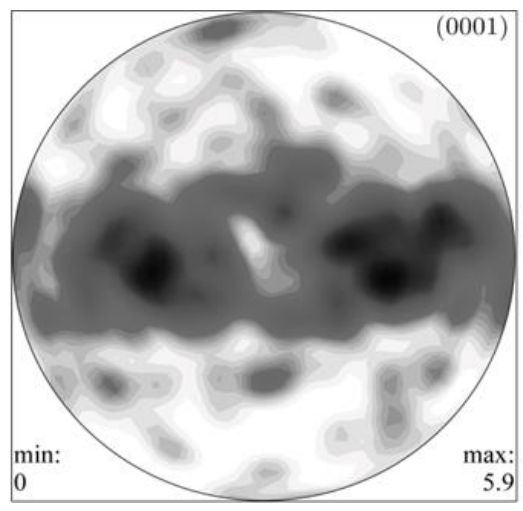

Fig. 9. The oligocrystal shown in (a) has been subjected to uniaxial strain in the Y-direction shown in (b) for a range of HCP textures shown in (c), (d) and (e). (c) represents a nominally random texture and two textures with preferred orientations are shown in (d) and (e). Note that the textures shown in (d) and (e) are opposite extremes such that in (d), the average c-axis lies approximately perpendicular to the loading direction and in (e), approximately parallel to the loading direction. 


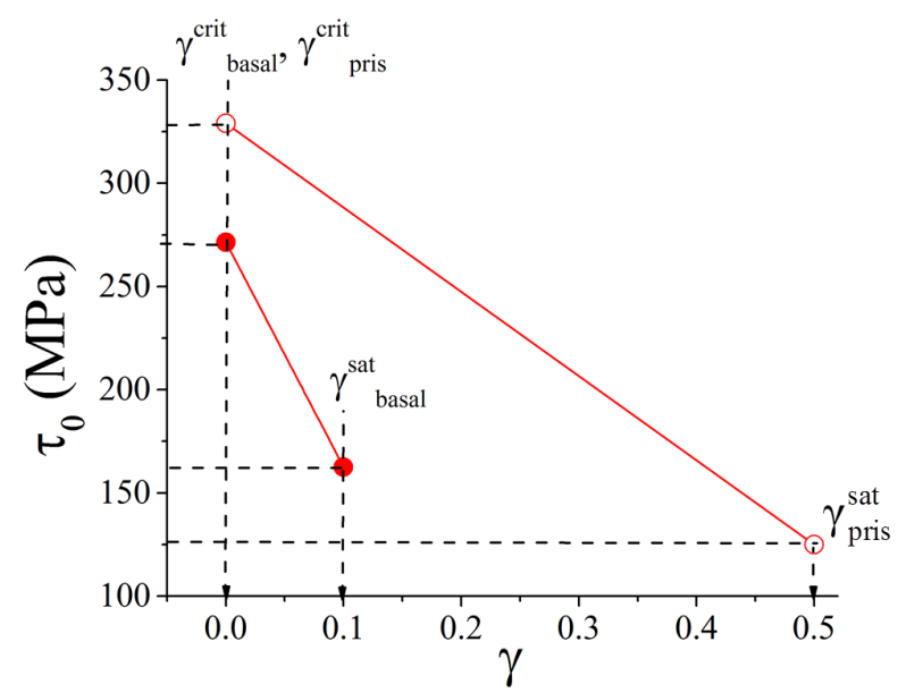

Fig. 10: Schematic of the softening regime applied on basal and prismatic slip systems respectively due to irradiation. The CRSS $\left(\boldsymbol{\tau}_{\mathbf{0}}\right)$ on both slip systems is reduced linearly from the irradiated to the unirradiated values upon achieving a critical level of slip on that individual slip system. In this example, the critical level is assumed to occur at the onset of slip on active slip systems i.e. $\boldsymbol{\gamma}_{\text {basal }}^{i}=\mathbf{0}$ and $\boldsymbol{\gamma}_{\text {pris }}^{i}=\mathbf{0}$. The rate of softening for basal slip is approximately twice that for prismatic slip.

Figs. 11ai, bi and ci show the accumulated plastic strain fields in the unirradiated polycrystal for the range of textures considered such that softening does not occur on any slip system. Figs. 11aii, bii and cii represent the accumulated plastic strain field for the three textures in which basal softening is prescribed according to Fig. 10 at the onset of slip on active slip systems. Finally, Figs. 11aiii, biii and ciii show the accumulated plastic strain fields for the range of textures such that basal and prismatic softening is prescribed according to Fig. 10.

For all textures considered, localization is observed with the introduction of basal softening and becomes more pronounced when both basal and prismatic slip system softening are implemented. Although prismatic softening is introduced at a slower rate compared to basal softening, its effect is significant in slip localization as seen in Fig. 11aiii, biii and ciii for the corresponding textures. This can be attributed to the larger range over which $\tau_{0}^{\text {pris }}$ decreases from the irradiated to the unirradiated values during softening. The corresponding mechanical stress response of each polycrystal texture is considered next.

Fig. 12 compares the mechanical stress response of the nominally random texture (Texture-A in Fig. 9c) with Texture-B. Note that Texture-B has a preferred c-axis orientation in the Xdirection (Fig. 9d), which is normal to the applied straining direction. In Fig. 12, three examples are presented, one in which softening does not occur on any slip system (termed no softening) one for which softening is introduced on the basal system (denoted basal softening only) and finally one for which basal and prismatic slip system softening are both included (denoted basal and prismatic softening). 


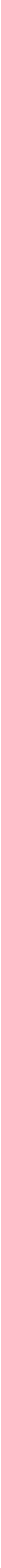


First consider no softening for the nominally random texture (Texture-A) and Texture-B in Fig. 12. The mechanical response indicates that the material yields and slips on favorably oriented slip systems leading to pronounced localization and a consequent drop in stress with increasing strain. In both textures, the onset of pronounced localization observed in the macroscopic stress-strain response begins at approximately 15 percent uniaxial strain. However, this is very much delayed compared to the response seen by introducing basal and prismatic softening. For Texture-B, the representative caxis direction in this texture is approximately orientated in the X-direction (Fig. 9d) leading to prismatic slip systems more favorably oriented for slip. Thus, introducing basal softening only has only small effect on the macroscopic mechanical response because slip is accommodated predominantly by prismatic systems, and, with the prismatic systems having a higher CRSS, localization is very much delayed leading to its absence in the average stress response observed. The addition of the introduction of prismatic softening to this texture leads to a much reduced flow stress shown in Fig. 12, together with the very rapid development of localization which manifests itself by the overall stress drop shown in Fig. 12.

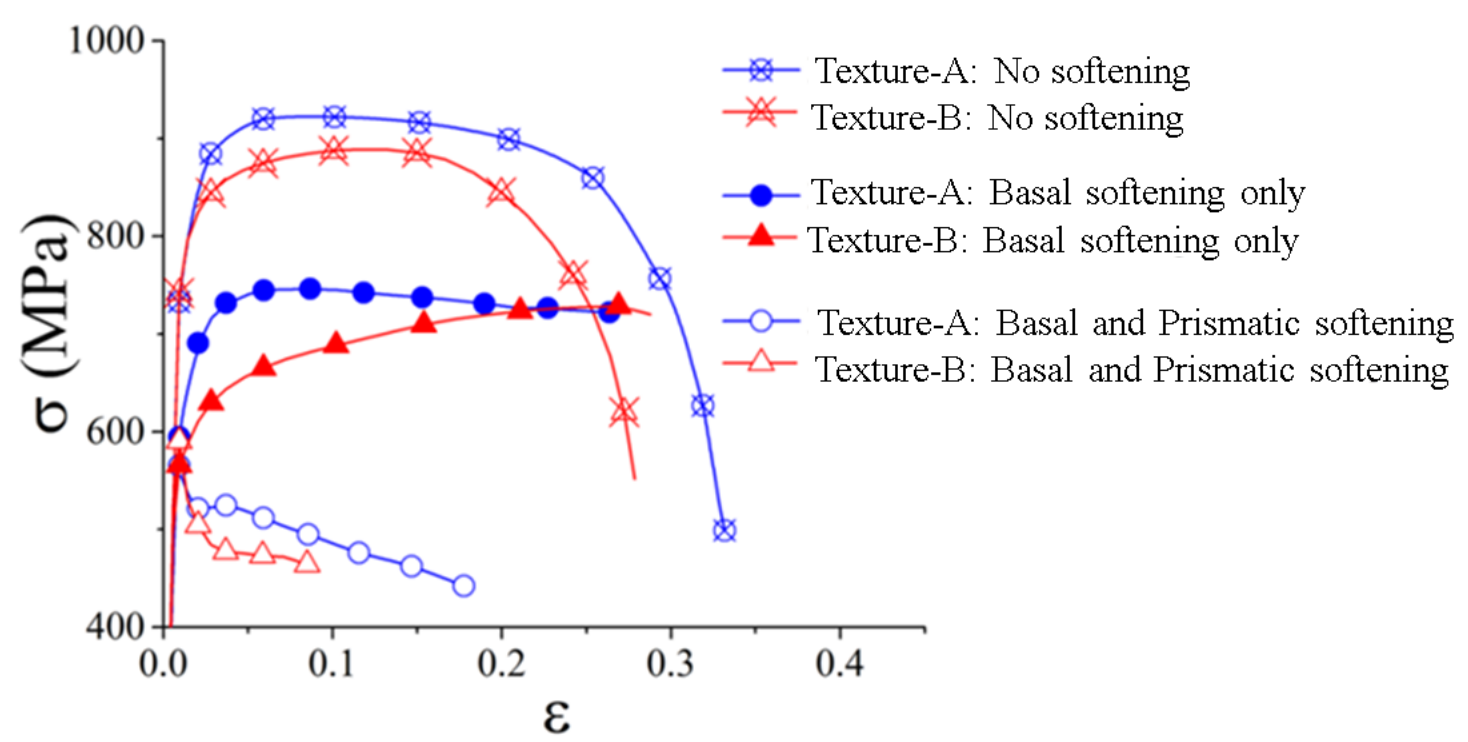

Fig. 12: The macroscopic $\sigma-\varepsilon$ curve for the nominally random and textured polycrystals (Texture-B shown in Fig. 9d)

The mechanical response for the nominally random texture (Texture-A) and Texture-C which has a preferred c-axis orientation approximately in the $\mathrm{Y}$-direction (parallel with the loading) is compared in Fig. 13. Similar to Fig. 12, the same three cases are presented. In the example in which no softening is allowed, the development of slip eventually leads to localization and the consequent drop in the macroscopic stress-strain response. Introducing basal softening and then prismatic softening in addition to basal softening leads to an earlier onset in the drop seen in the macroscopic stress response. Considering the introduction of basal softening only for Textures- B and C, the delayed onset of a marked decrease in the stress response to localization for Texture-B results from the preference for slip on the prismatic slip systems. However, for Texture-C (which is representative of textures used in nuclear reactor cladding materials), both basal and prismatic slip systems are easily favorably oriented for slip and this results in the relatively earlier onset of marked localization seen in the stress-strain response. 


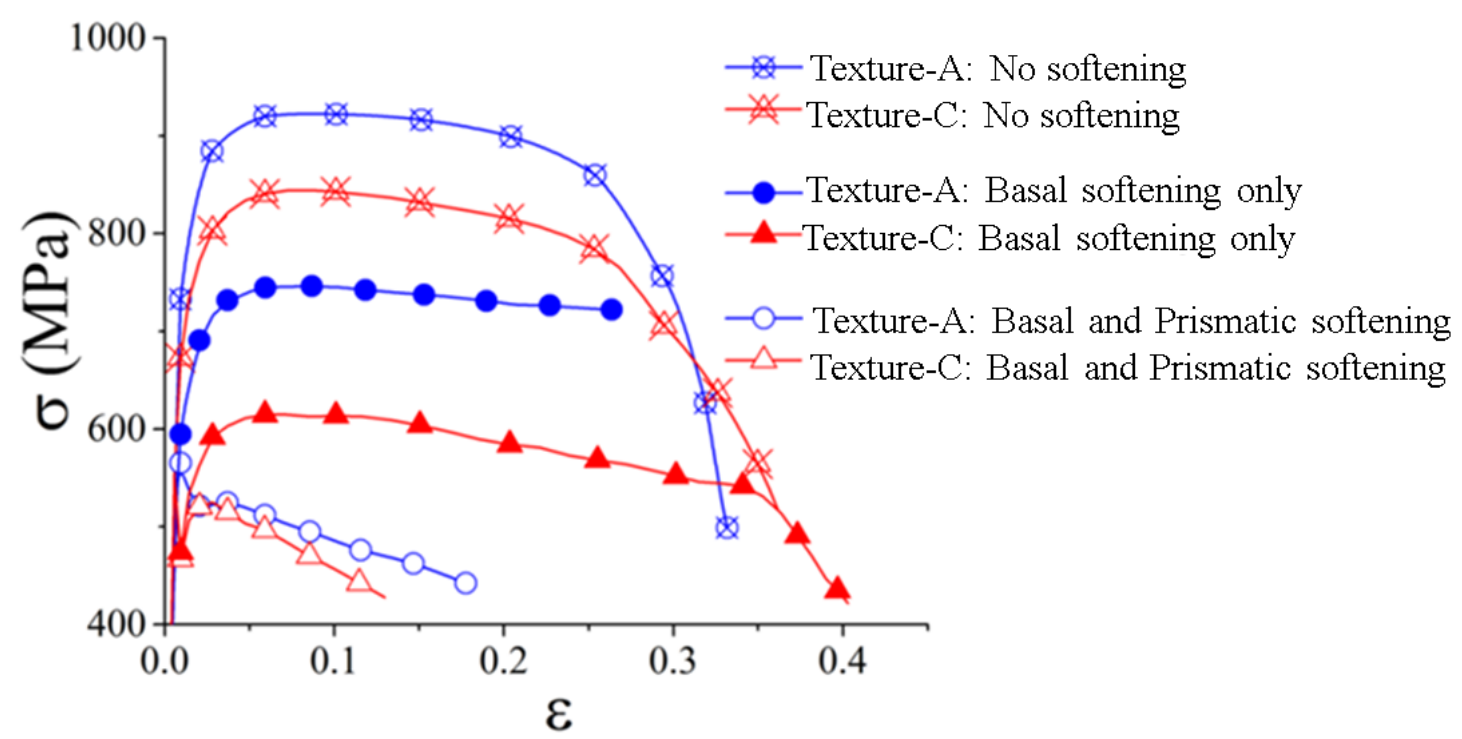

Fig. 13: The macroscopic $\sigma-\varepsilon$ curve for the nominally random (Texture-A) and textured polycrystals (Texture-C shown in Fig. 9e)

An analysis of the accumulated slip fields for individual slip systems for Texture-C shows that the initial development of slip bands occur predominantly on basal systems at an applied strain of about $1 \%$ which further consolidate and connect with neighboring slip bands with increasing strain. A much more pronounced activity is observed on the basal channels compared to the prismatics as a result of this particular texture, leading to pronounced localization on basal systems.

The studies presented so far indicate that the experimentally observed softening behaviour resulting from irradiation is unlikely to relate to basal slip systems alone, since this would preclude the rapid strain localization and stress decrease observed experimentally and shown in Fig. 1. This is emphasized by considering the marked effect of prismatic slip system softening on the average stress response shown in Figs. 12 and 13 in comparison to Fig. 1, and that which occurs for basal softening alone. The average stress responses calculated show that although basal softening contributes to a progressive decrease in the stress-strain response, the incorporation of prismatic softening is significant, and when considered together with basal softening, results in a response much closer to that observed in experiments. In fact, a systematic assessment of basal softening alone has been carried out, which includes the incorporation of high rate of basal softening, which shows that basal softening alone cannot lead to the capture of the experimental localization and softening response, and details are provided in Appendix D. It is therefore proposed that basal and prismatic softening are both required to replicate the experimentally observed mechanical response.

In the previous example, softening was prescribed at a lower rate on the prismatic compared to basal slip system seen in Fig. 10, and at the onset of slip. The rates which occur experimentally and in practice are, of course, currently unknown; hence, focus is now placed on calibrating the softening introduced on basal and prismatic slip systems in order to replicate the experimentally observed mechanical response shown in Fig. 10. It is assumed that there exists a critical point at which softening is initiated leading to accelerated localization. On this basis, softening is prescribed at the 
same rate on both basal and prismatic slip systems and is assumed to commence at the same value of critical slip as illustrated in Fig. 14.

Fig. 15 shows Texture-C subjected to uniaxial straining for cases in which no softening is prescribed on basal or prismatic slip systems, and shows two cases in which slip system softening is prescribed. First, the softening is prescribed at the onset of slip on individual slip systems and in the second example, a critical value $\left(\gamma^{\text {crit }}=0.06\right.$ ) is chosen to represent the onset of softening. A relatively flat stress response is observed in the example with no softening incorporated; however, a marked drop characteristic of the experimental mechanical response of irradiated materials is observed with the incorporation of softening. A pronounced drop is seen when softening is prescribed at the onset of $\operatorname{slip}\left(\gamma^{\text {crit }}=0\right)$ but a better representation of the experimentally measured stress strain response (Fig. 1) presented by Wei et al. [4] is observed when a critical value for softening is adopted ( $\gamma^{\text {crit }}=$ 0.06). Note that the texture considered by [4] is only similar to Texture-C. Hence, it is therefore hypothesised that irradiation softening affects both basal and prismatic slip systems and that a critical slip point exists at which softening is triggered. In this way, the experimentally observed localization resulting from slip system softening can be reasonably reproduced; however, the incorporation of basal slip system softening alone does not enable a good reproduction of experimental results, as demonstrated in Appendix D. Unfortunately, experimental quantification of the rates of softening for both basal and prismatic slip systems remain elusive, but the present study at least enables the onset of softening and the (assumed linear) rate of softening to be evaluated. As a result, less emphasis is placed here on direct calibration of the model for the experimental behaviour shown in Fig. 15. Rather, we assess the relative contributions from both basal and prismatic slip softening in order to establish their role in affecting the experimentally observed localisation and failure.

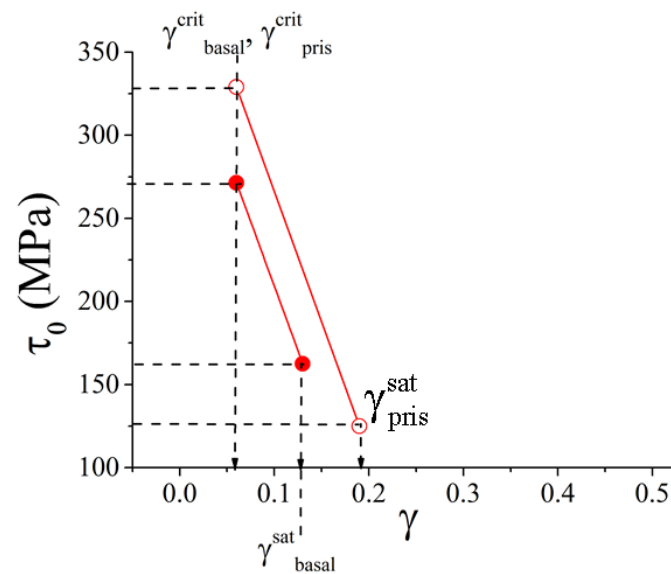

Fig. 14: Schematic of the softening regime applied on basal and prismatic slip systems due to irradiation. The CRSS on both slip systems is progressively reduced from the irradiated to the unirradiated values upon achieving a critical level of slip on that individual slip system. 


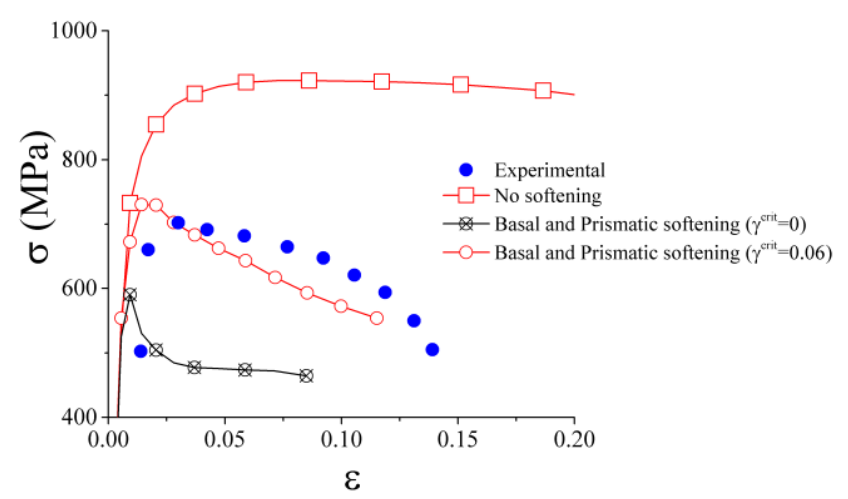

Fig. 15: The macroscopic $\boldsymbol{\sigma}-\boldsymbol{\varepsilon}$ curve for the textured polycrystal (Texture-C) subjected to uniaxial strain in the Y-direction. The unirradiated case (no softening) is compared with the irradiated case. Two examples of the irradiated case are shown such that basal and prismatic softening are initiated at the onset of slip versus the case where softening is initiated at a critical value of slip.

Using the coupled basal and prismatic system softening model so established, the paper next addresses more representative in-service loading conditions and resultant localization and failure conditions.

\section{Polycrystal zircaloy subjected cyclic loading}

In-service materials are often subjected to continuous cycles, and for zircaloy typically at smaller magnitude load levels than those considered so far, but potentially leading to significant stress localization and eventually failure. Cyclic loads can be either strain controlled or stress controlled and in this study, for completeness, both forms of loading are considered.

The oligocrystal shown in Fig. 9a is subjected to cyclic loading and focus is placed on Texture-C (Fig. 9e) which is representative of nuclear reactor cladding material. Also, from the previous section, it has been established that both basal and prismatic softening must be activated at a critical level of slip $\left(\gamma^{\text {crit }}=0.06\right)$ in order to achieve the experimentally observed mechanical response. These properties have been adopted and the oligocrystal is subjected to reversed stress and strain controlled cyclic loading.

\subsection{Strain controlled cyclic loading}

The oligocrystal in Fig. 9 incorporating Texture-C was subjected to 10 cycles of $R=-1$ strain controlled cyclic loading with peak strain of $1 \%$ illustrated in Fig. 16. Tensile strain was applied in the Y-direction followed by unloading, then similarly, compressive straining in the Y-direction followed by unloading. This process was repeated for 10 cycles and the resulting stress-strain history is obtained for two examples, one in which no basal nor prismatic softening is implemented and a second in which basal and prismatic softening are both activated at the critical level of slip. 


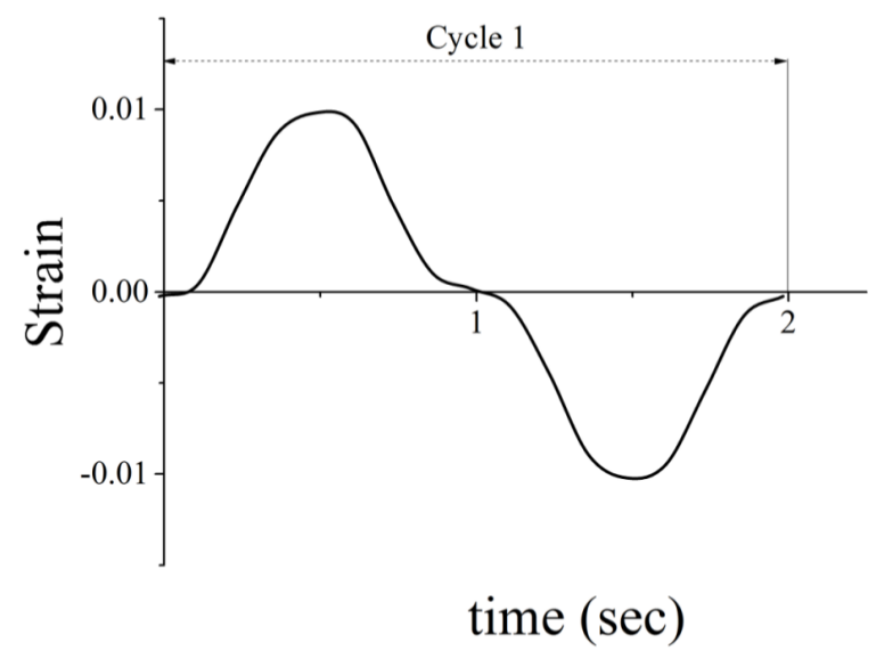

Fig.16: Schematic of the fully reversed strain-controlled cyclic loading history $(R=-1)$

The effects of basal and prismatic slip system softening are evident in the stress strain response seen in Fig. 17. Softening leads to the progressive relaxation in the achieved macroscopic stress-strain response in the polycrystal with increasing numbers of cycles. The accumulated slip fields for the two examples considered are shown in Fig. 18. The slip fields are shown for selected cycles (1, 5 and 10) in order to investigate the formation of localized slip bands. The example with basal and prismatic softening indicates the progressive development and growth of localized slip bands with increasing number of cycles, with accumulated plastic strains of $\sim 100 \%$. No such localization develops in the absence of the slip system softening. Studies by Coleman et al. [25] on irradiated zircaloys indicate that the local levels of plastic strain can approach $100 \%$.

Fig. 19 provides a further analysis of the accumulated slip fields shown in Fig. 18. Fig. 19a shows the Y-Z plane of the accumulated slip field shown in Fig. 18c (without softening) and Fig. 19b shows the example where basal and prismatic softening is implemented (Fig. 18f). Considering Fig. 19b, it is evident from the schematic showing the typical grain size in the model that the development of slip bands grows across many grains. The manifestation of this is seen in Fig. 19d which shows the accumulated slip at two points indicated on Fig. 19a. It is clear that both elements (A and B) show differing levels of slip. Element B lies within a localized slip band leading to much higher slip level compared to Element A. The wavy response observed in the accumulated slip developed with increasing number of cycles is examined in the inset of Fig. 19c which shows the progressive development of slip during loading reflecting its cyclic nature.

Considering further Fig. 19b, the introduction of basal and prismatic softening leads to the establishment and intersection of localized slip bands, marked by the dashed lines, giving rise to high levels of accumulated slip illustrated for Elements A and B in Fig. 19d. The location of the slip band is argued to be of significance according to studies by Adamson et al. [7]. The Y-Z section shown lies on the surface of the polycrystal and [7] indicates that slip bands intersecting with the surface increases susceptibility to stress corrosion cracking in zircaloys used as cladding material in pressure water reactors [7]. A further analysis of Element A shown in Fig. 20 reveals that it lies within a grain which has the crystallographic orientation shown in Fig. 20a such that its c-axis is orientated at about $50^{\circ}$ to the straining direction and is favourably oriented for both basal and prismatic slip. 
Other locations within the polycrystal at which slip bands have initiated are also evaluated with the local orientation as well as the basal and prismatic Schmid factors detailed in Table 2. It is seen from Table 2 for all locations considered that both basal and prismatic slip are favoured at the nucleation site and that in all cases considered, both basal and prismatic Schmid factors are reasonably high ( 0.2 and higher).

Table 2: Local orientation details for grains at the initiation of slip bands

\begin{tabular}{|l|c||c||c||c||}
\hline Location & A & B & C & D \\
\hline $\begin{array}{l}\text { C-axis orientation w.r.t. loading direction } \mathbf{( 2 -} \\
\text { direction) }\end{array}$ & $80^{0}$ & $59^{0}$ & $50^{0}$ & $36^{0}$ \\
\hline Basal Schmid factor & 0.18 & 0.42 & 0.45 & 0.4 \\
\hline Prismatic Schmid factor & 0.4 & 0.33 & 0.23 & 0.2 \\
\hline
\end{tabular}

It should be noted however that although these sites within the polycrystal may be favourable for slip on both basal and prismatic systems, it does not necessarily implicate the development of a slip band. It is therefore hypothesized that neighbourhood effects play a role in slip band growth. To analyse this, a slip band which grows across a number of grains is analysed to evaluate the role of neighbouring grains on slip band growth.

Two examples are considered to evaluate neighbourhood effects and the details of the slip bands are presented in Table 3. Table 3 shows the c-axis orientation for grains that lie within a slip band. It is seen in both examples considered that the grains have similar crystallographic configurations, are favourably oriented for both basal and prismatic slip and that the Schmid factor on both systems are considerably high $(\sim 0.28$ and higher).

Table 3: Local orientation details for grains within a slip band

\begin{tabular}{|c|c|c|c|}
\hline & C-axis orientation w.r.t. loading direction (2-direction) \\
\hline \hline & Grain 1 & Grain 2 & Grain 3 \\
\hline \hline Example 1 & $57^{0}$ & $62^{0}$ & $62^{0}$ \\
\hline \hline Example 2 & $61^{0}$ & $57^{0}$ & $51^{0}$ \\
\hline
\end{tabular}

It is therefore postulated that slip band nucleation occurs in grains highly favouring basal and prismatic slip and that growth occurs if neighbouring other grains of similar crystallographic orientation.

\subsection{Stress controlled cyclic loading}

A fully reversed stress cycle $\left(\sigma_{\mathrm{YY}}=500 \mathrm{MPa}\right)$ illustrated in Fig. 21 is applied in the Y-direction of the oligocrystal shown in Fig. 9a with Texture-C incorporated. The magnitude of applied stress was carefully selected to achieve slip locally within grains favorably oriented. Therefore, whilst the average macroscopic stress strain response of the polycrystal may indicate an elastic behavior, stresses found locally within the polycrystal in grains favorably oriented for slip can be sufficiently high to activate slip.

Consider Fig. 22 which shows the macroscopic stress-strain response for the oligocrystal shown in Fig. 9a subjected to 10 cycles of stress controlled cyclic loading. As before, two examples are considered. Fig. 22a shows the macroscopic response without basal and prismatic softening while Fig. 22b shows the stress-strain response with softening included on the basal and prismatic slip 
systems upon achieving a critical level of slip $\left(\gamma^{\text {crit }}=0.06\right)$. The effect of basal and prismatic softening is immediately evident due to the loop broadening behaviour observed in Fig. $22 b$ compared to Fig. 22a. A closer analysis, reflected in Fig. 22b, indicates a progressive increase in the accumulated strain resulting from localized slip bands formed within the polycrystal. This is illustrated in Fig. 23 which shows the average accumulated slip developed within the polycrystal with increasing number of cycles, which demonstrates a significant consequence of the development of slip bands is the establishment of structural ratcheting, potentially leading to macroscale necking and component failure. In Fig. 23, the early response for both examples is the same, but at higher cycles, once the softening criterion is progressively achieved at differing locations in the polycrystal, the consequent ratcheting drives localization leading to the significant differences observed.

Fig. 24 further emphasizes the accumulation of slip in the two examples considered in Fig. 22 after 10 cycles of loading. It is evident from Fig. 24 that the introduction of basal and prismatic softening leads to clearly defined localized slip bands which develop and grow with increasing number of cycles. A further analysis of the development of slip bands is examined in Fig. 25 which shows the accumulated slip between cycles 5 and 10 for the example with basal and prismatic softening shown in Fig. 24. Slip band formation, indicated by the dashed lines, initiates during cycle 5, growing progressively with increasing numbers of cycles. Subsequently, localized slip bands begin to form in other favorably oriented grains leading to growth and eventual intersection with already existing slip bands.

The fully-reversed cyclic loading (with zero mean stress) is not entirely characteristic of in-service loading conditions. In-service loading often gives rise to non-zero mean stress cyclic loading regimes such as that shown in Fig. 26 which consists of continuous cycles of loading and unloading as shown and Fig. 27 shows the macroscopic stress-strain response for the two examples considered. Fig. 27a shows the polycrystal response without basal or prismatic softening and shows modest ratcheting of the stress-strain response with increasing number of cycles, and that plastic shakedown occurs leading to steady-state ratcheting after just two cycles. Fig. 27b, however, shows the result for which basal and prismatic softening are implemented at the critical level of slip and it is evident that softening leads to significant ratcheting seen in the stress-strain response, and that the ratcheting rate is influenced strongly by the presence of the applied non-zero mean stress. The ratcheting behaviour is attributed to the development of localized slip within the polycrystal leading to the significant levels of plastic ratchet strains illustrated by Fig. 28, which shows the average accumulated slip for the two cases considered. Following the first cycle in Fig. $28(0.5<\mathrm{N}<1)$, the softening criterion promotes localization causing an increase in the average accumulated slip within the polycrystal. The average slip stabilizes initially as seen by the plateau in the figure with softening and then increases significantly thereafter. This results from the onset of localization in other favorably oriented grains, slip band growth, and the subsequent interaction and coalescence of slip bands leading to the observed response. 
(a)

No Softening

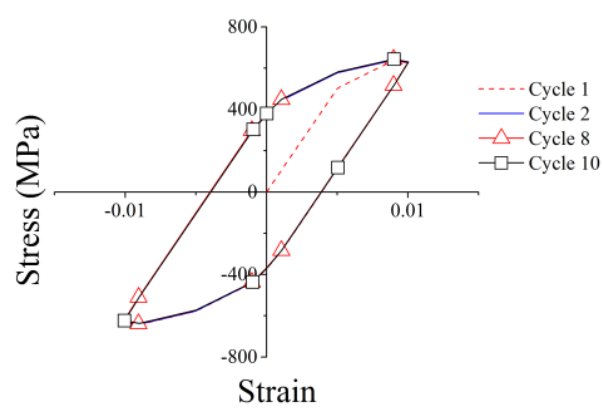

(b)

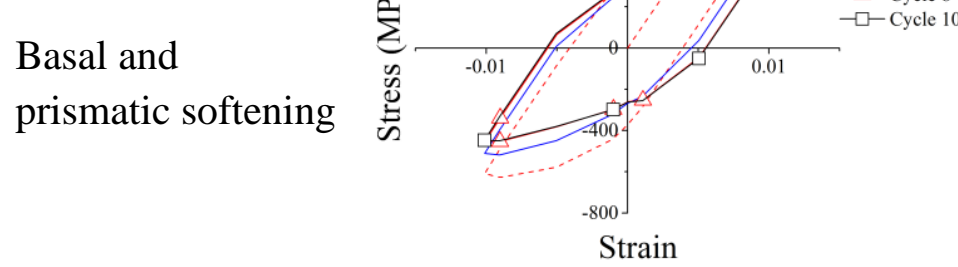

Fig. 17: Macroscopic stress-strain loops for initially textured polycrystal (Texture-C) subjected to 10 cycles of fully reversed straincontrolled cyclic loading $(R=-1)$. The example with no softening on basal and prismatic slip systems is shown in (a) and the loops generated upon introduction of softening shown in (b). 


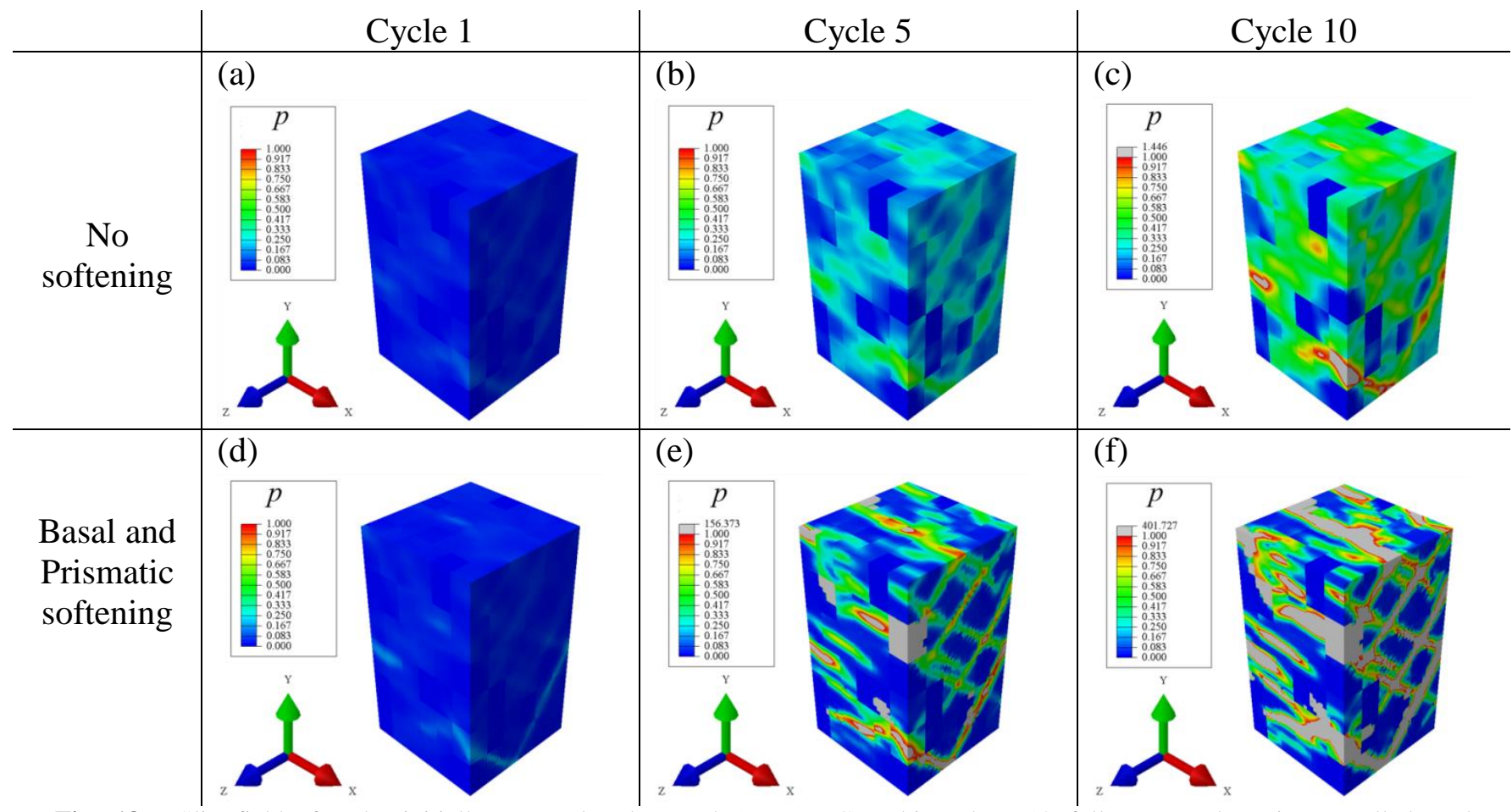

Fig. 18: Slip fields for the initially textured polycrystal (Texture-C) subjected to 10 fully reversed strain-controlled cycles (macroscopic stress-strain data presented in Fig. 17), shown for cycles 1, 5 and 10 for no softening in (a), (b), (c) and when softening is introduced on the basal and prismatic slip systems, shown in (d), (e) and (f).

(a)

\section{No Softening}

(b)

\section{Basal and prismatic softening}

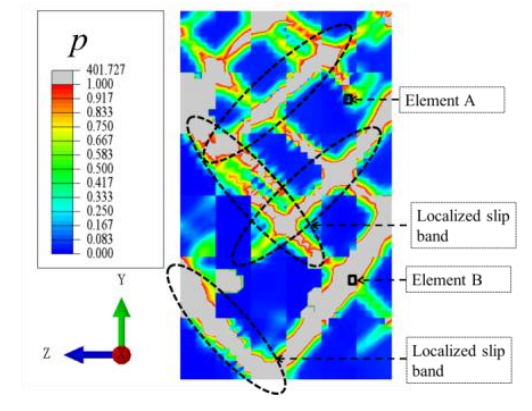

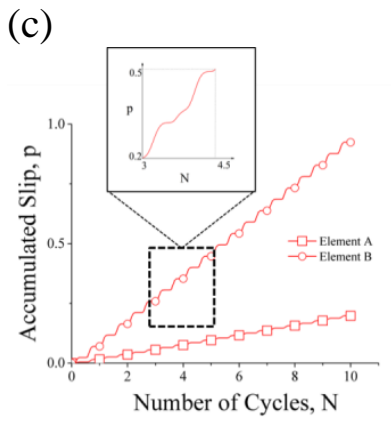

(d)

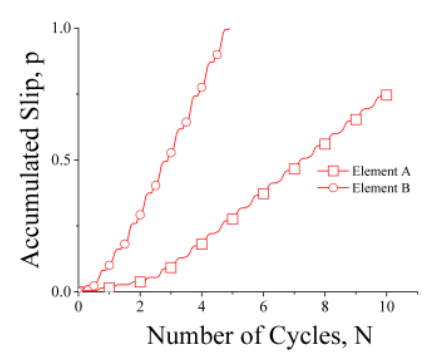

Fig. 19: Analysis of the accumulated slip developed at Cycle 10 in Fig. 18. The accumulated slip in the Y-Z plane is shown in (a) and (b) for the irradiated example without softening and with softening respectively. The accumulated slip is further shown at particular points (Elements A and B) in both examples shown in Fig. (c) and (d) for the case without softening and with the incorporation of basal and prismatic softening respectively. 
(a)

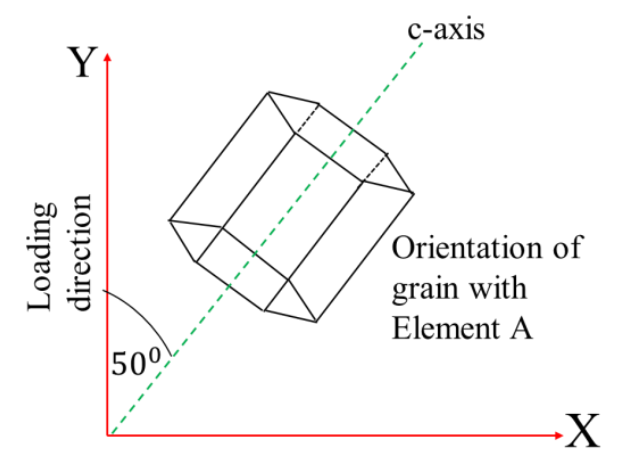

(b)

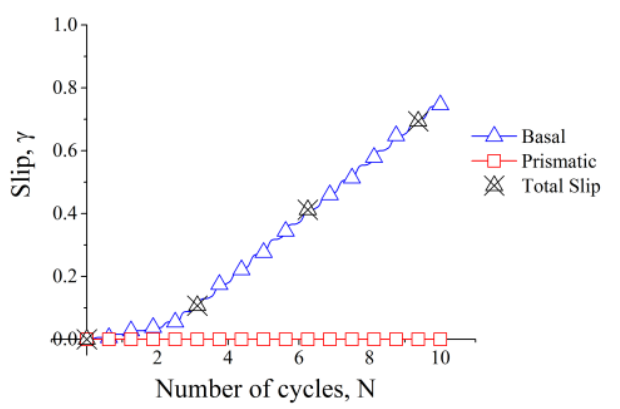

Fig. 20: Analysis of the slip developed in Element A shown in Fig. 19. (a) shows the crystallographic orientation favorable for basal and prismatic slip and (b) shows the accumulated slip on basal and prismatic systems.

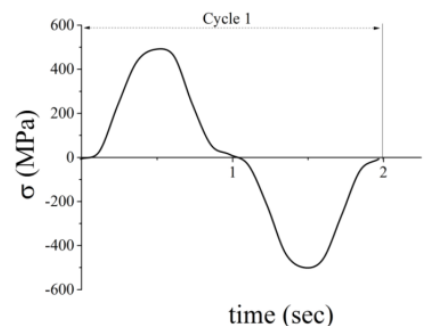

Fig. 21: Schematic of the fully reversed stress-controlled cyclic loading history $(R=-1)$ 
(a)

No Softening

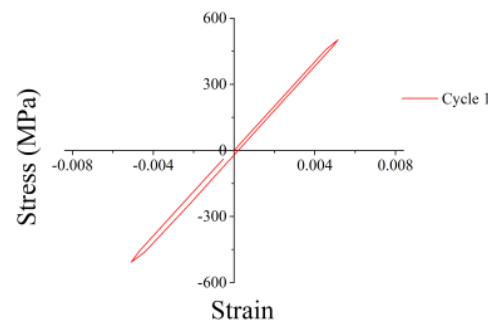

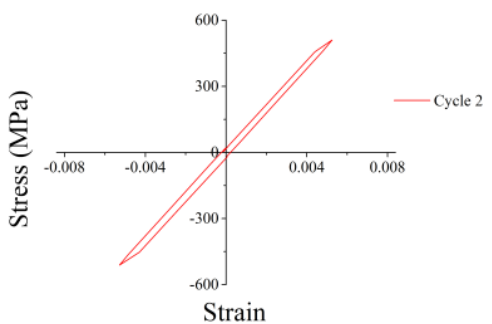
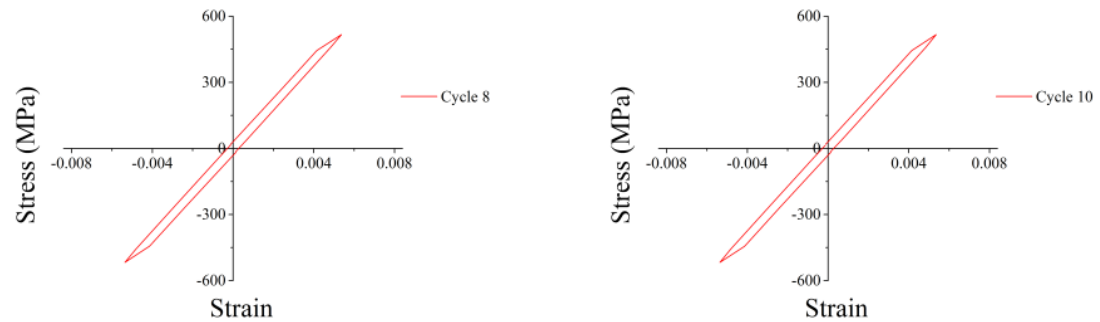

(b)
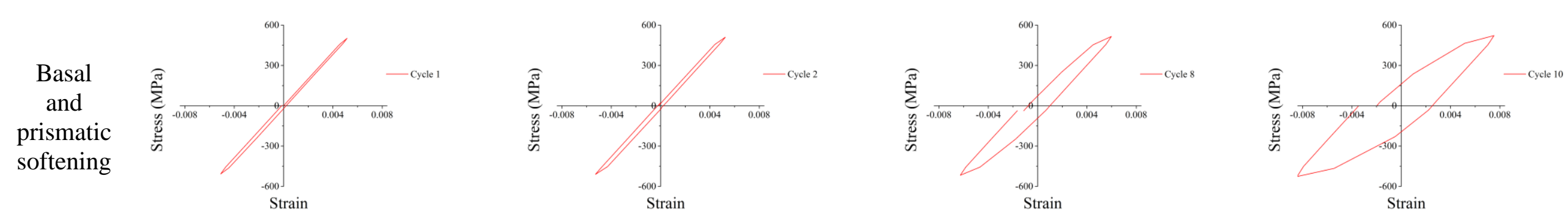

Fig. 22: Macroscopic mechanical response for initially textured polycrystal (Texture-C) subjected to 10 cycles of fully reversed stress-controlled cyclic loading ( $R=-1)$. The stress-strain response for the case without softening on basal and prismatic slip systems is shown in (a) and the loops generated with softening are shown in (b). Only small cyclic changes are observed in (a) with rapid shakedown, which is not the case with the introduction of softening seen in (b). 


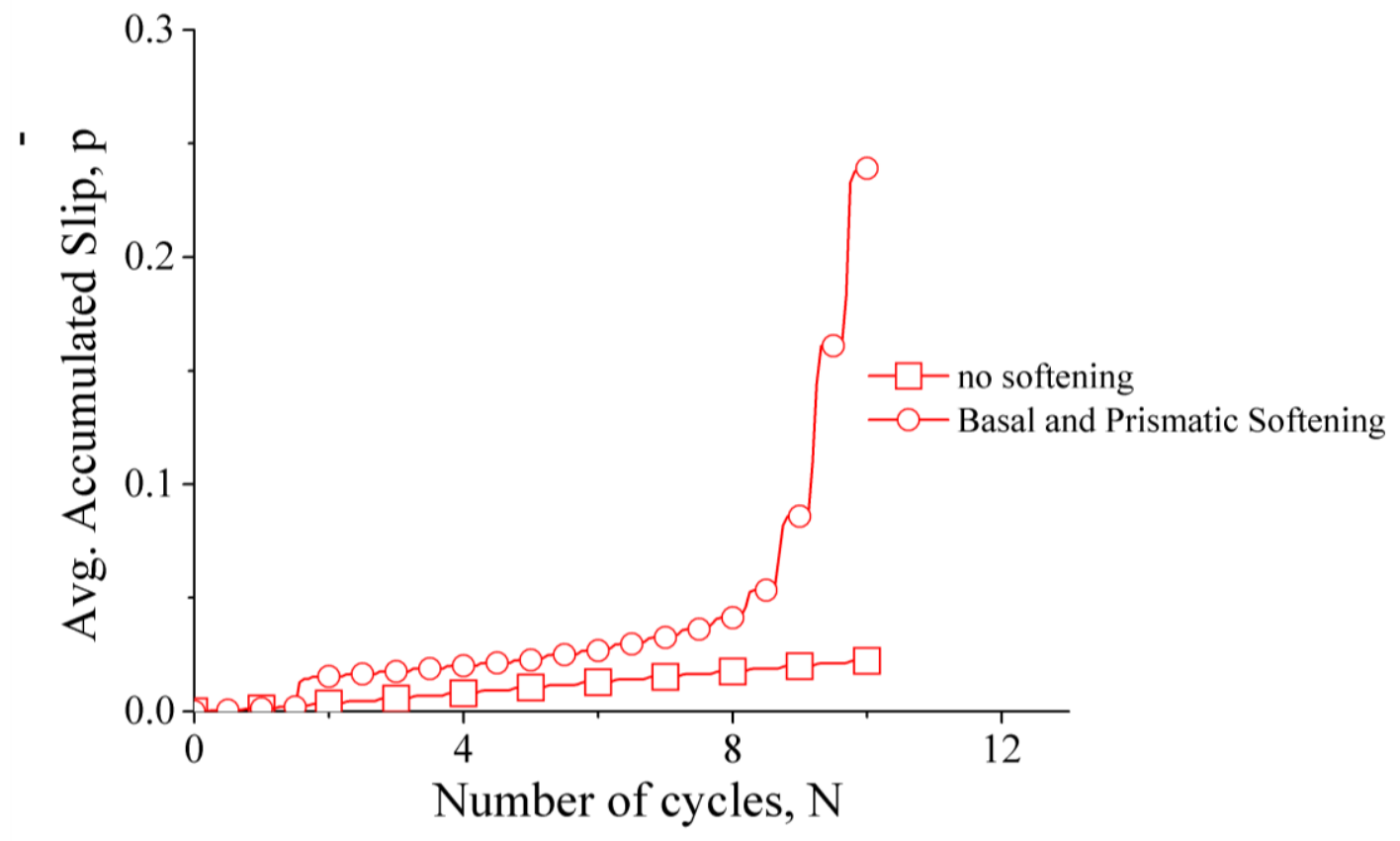

Fig. 23: Average accumulated slip developed for 10 cycles of fully reversed stress controlled cyclic loading

(a)

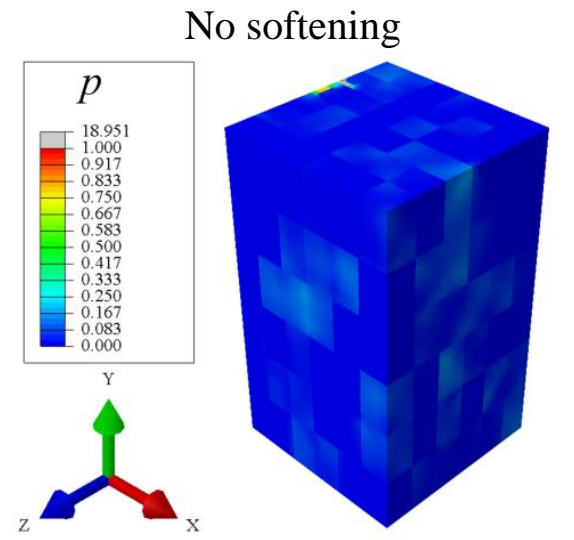

(b)
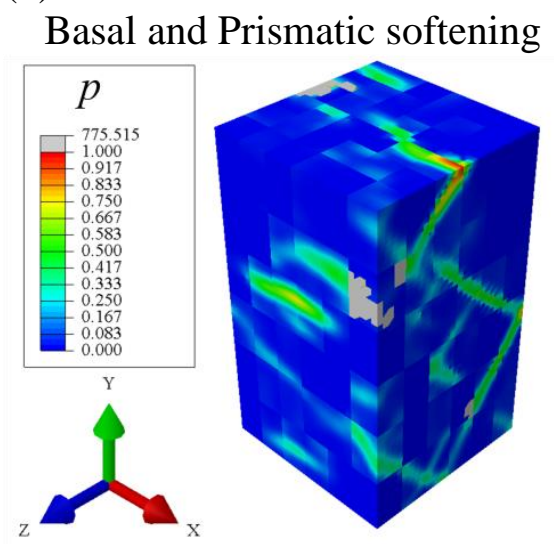

Fig. 24: Slip field for the initially textured polycrystal (Texture-C) subjected to 10 fully reversed stress-controlled cycles. The slip fields are shown after Cycle 10 for the example with no softening shown in (a) and when softening is introduced on the basal and prismatic slip systems shown in (b). Localized slip bands form with increasing number of cycles and become more pronounced when softening is incorporated leading to the ratcheting observed in the macroscopic stress-strain response seen in Fig. 23. 


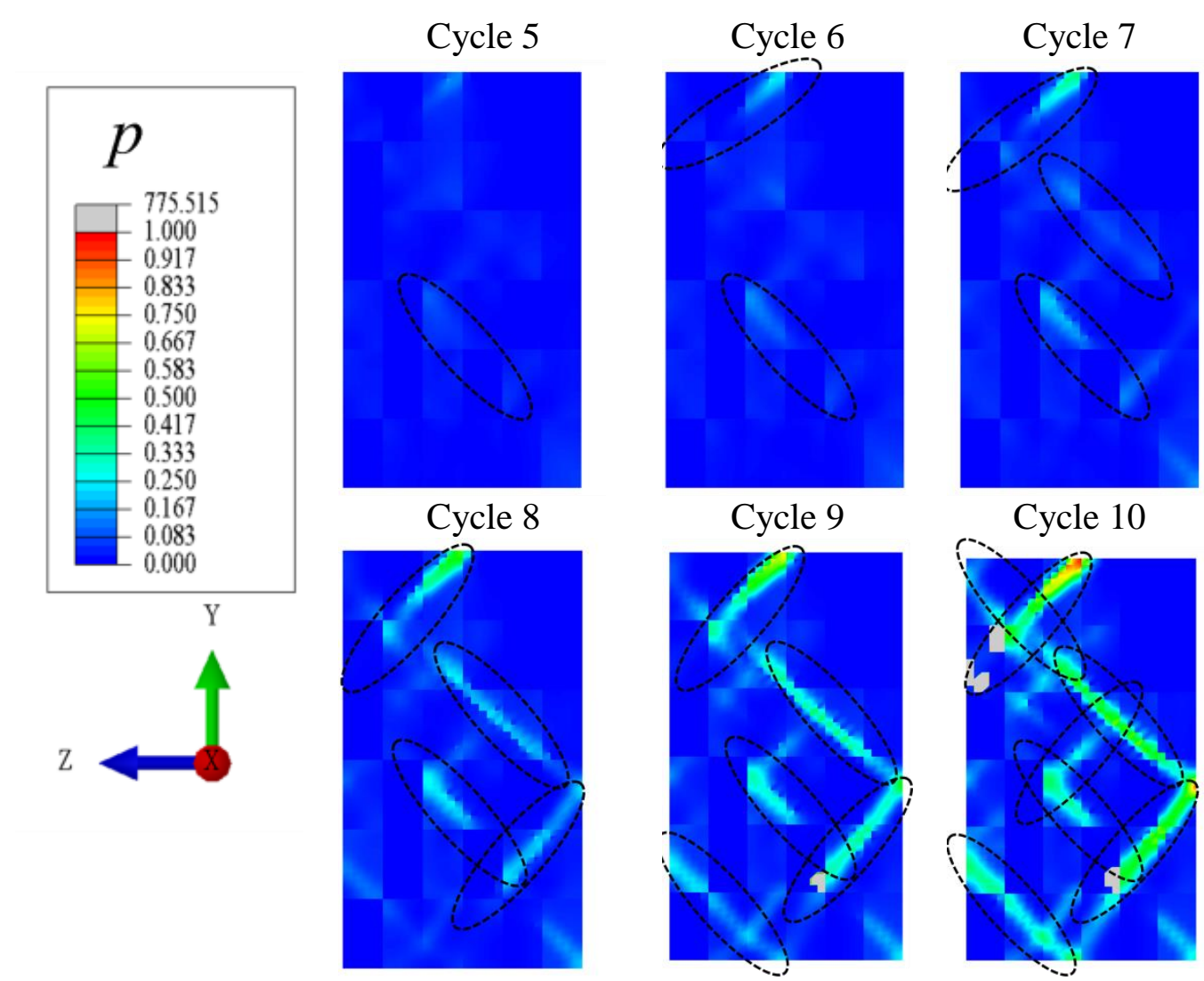

Fig. 25: Progressive development of localised slip bands upon the introduction of softening in the irradiated material. The slip bands developing during cycle 5 grow and become more pronounced at higher cycles. Note the consequent intersection of neighbouring slip bands indicated by the dashed lines.

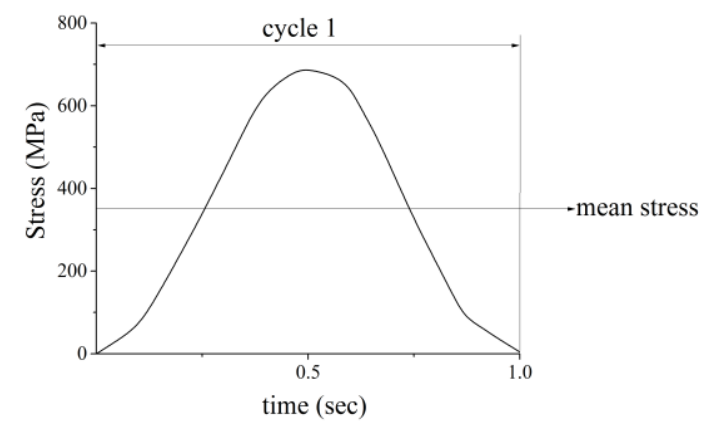

Fig. 26: Schematic of the stress-controlled cyclic loading history $(R=0)$ 
(a)

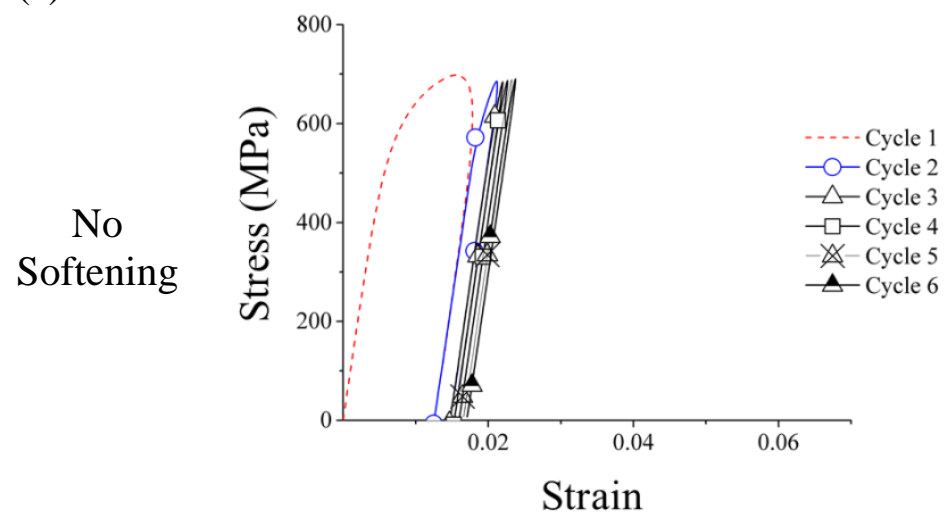

(b)

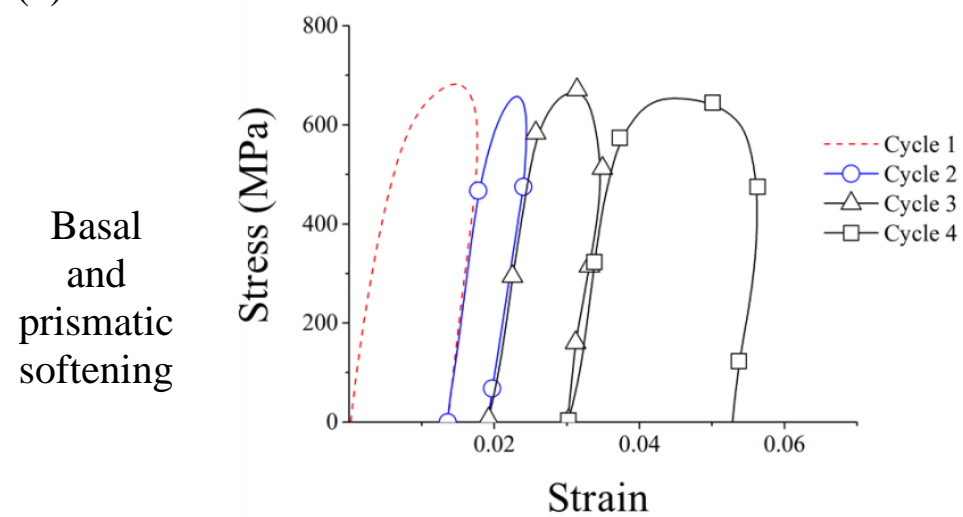

Fig. 27: Macroscopic mechanical response for initially textured polycrystal (Texture-C) subjected to 4 cycles of stresscontrolled cyclic loading $(R=0)$. The stress-strain response for the case without softening on basal and prismatic slip systems is shown in (a) and the loops generated upon introduction of softening are shown in (b).

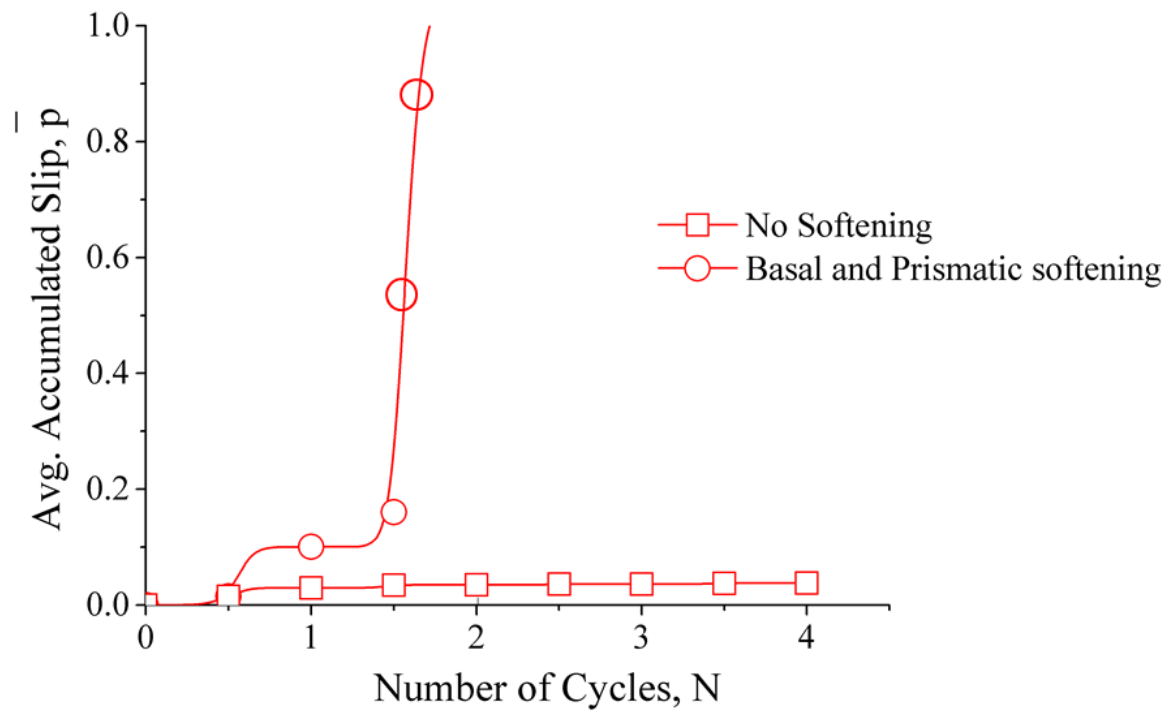

Fig. 28: Average accumulated slip developed for 4 cycles of stress controlled $(R=0)$ cyclic loading. 


\subsection{Effects of GND density}

The GND density methodology described in section 2.1 is used to obtain GND densities presented in this section. The contribution of GND density to the slip strength is calculated using Taylor's approach such that

$$
\tau=\tau_{0}+G b \sqrt{\rho_{\mathrm{GND}}+\rho_{\mathrm{SSD}}} .
$$

Assuming $\rho_{\mathrm{SSD}}$ is constant, the contribution of GNDs to the slip strength can be estimated using

$\tau=\tau_{0}+G b \sqrt{\rho_{\mathrm{GND}}}$

where $G=33 \mathrm{GPa}$ is the shear modulus and $b=2.95$ Angstroms is the Burgers vector.

To illustrate the role of GND evolution, its effect is evaluated for two examples; (1) The irradiated polycrystal in Fig. 11 and (2), the example of the polycrystal under non-zero mean stress-controlled cyclic loading which showed pronounced ratcheting (Fig. 27).

The GND calculation given in Fig. 29 shows the density of GNDs in the irradiated polycrystal under uniaxial straining at 5 percent presented in Fig. 11ciii. At this level of macroscopic strain, the localized softening is already having an effect on the macroscopic stress strain response seen in Fig. 13 (Texture C) and localized slip bands are already developing as indicated. Using the Taylor relation described above and adopting the maximum density of GND within the polycrystal, it is seen that the maximum contribution to slip strength due to GNDs $\left(G b \sqrt{\rho_{\mathrm{GND}}}\right)$ is $\tau=\tau_{0}+30 \mathrm{MPa}$ which is small compared to the irradiated slip system strengths on basal and prismatic systems which are $271.5 \mathrm{MPa}$ and $329 \mathrm{MPa}$ respectively as well as the irradiation induced reductions in strength which are $109 \mathrm{MPa}$ and $204 \mathrm{MPa}$ for the basal and prismatic systems respectively. Also, local to a slip band shown in Fig. 29, the contribution of GNDs is $\tau=\tau_{0}+17 \mathrm{MPa}$.
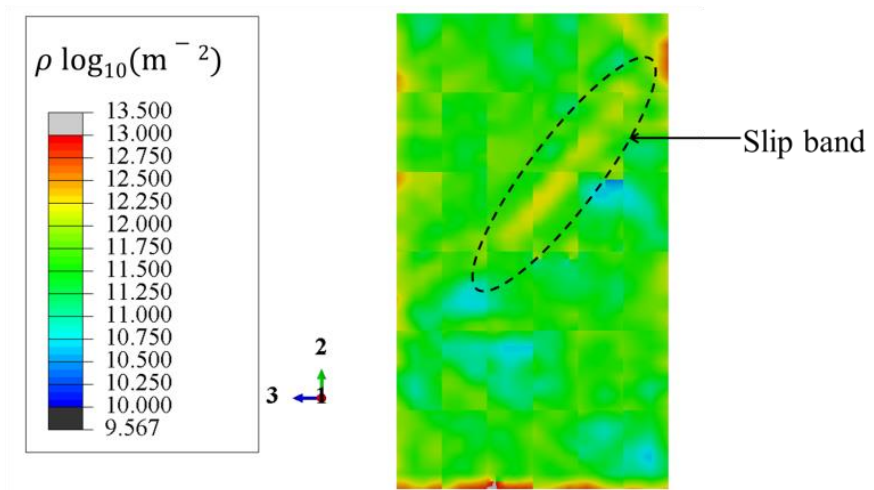

Fig. 29: Development of GNDs in polycrystal (Texture C) subjected to uniaxial straining at 5 percent strain indicating a localized slip band

The second example considered is the case of non-zero, mean stress-controlled cyclic loading which showed pronounced ratcheting. A similar analysis on the contribution of GNDs to slip strength as presented above is considered for the four cycles presented in Fig. 27 using the maximum GND density developed locally. First, an evolution of GNDs is presented in Fig. 
30 at the peak stress for four non-zero mean stress cycles and subsequently, a table which uses the maximum GND density to calculate the potential contribution to slip strength is presented.
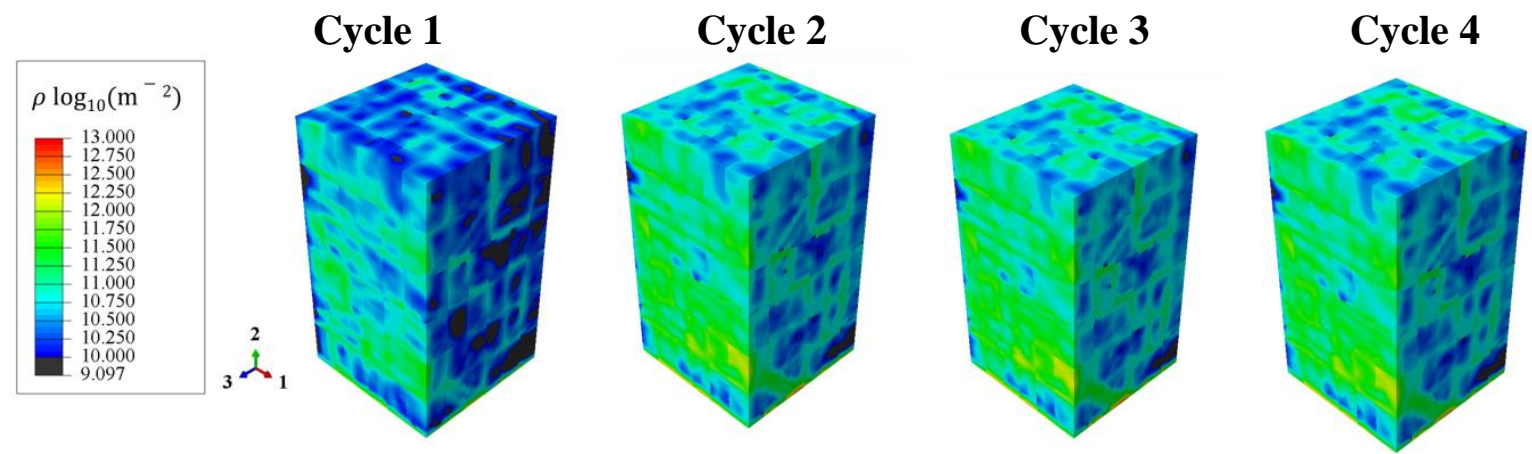

Fig. 30: Evolution of GNDs in non-zero mean stress-controlled cyclic loading

Table 4: The progressive development of GNDs with increasing number of cycles and relative contribution to slip strength

\begin{tabular}{|c|c|c|c|c||}
\hline Cycle & $\mathbf{1}$ & $\mathbf{2}$ & $\mathbf{3}$ & $\mathbf{4}$ \\
\hline \hline$\rho_{\mathrm{GND}}^{\mathrm{max}}\left(\log _{10} \mathrm{~m}^{-2}\right)$ & 9.1 & 12.9 & 13.0 & 13.02 \\
\hline \hline Contribution to slip strength, $G b \sqrt{\rho_{\mathrm{GND}}}(\mathrm{MPa})$ & 0.3 & 23.3 & 30 & 31.5 \\
\hline \hline
\end{tabular}

It becomes apparent from Fig. 30 and Table 4 that whilst accounting for GND density is important, it does not strongly affect the macroscopic response as the effect of softening dominates any small-scale hardening due to GND development. It is therefore hypothesized that whilst GNDs may diminish the rate of localization observed, the rate of irradiation induced slip system softening likely dominates macroscopic mechanical behaviour.

\section{Conclusions}

Single crystal studies on zircaloy using crystal plasticity have shown that irradiation-induced softening affecting only basal slip leads to the establishment of slip localization and shear band formation. However, polycrystal studies show that the influence of softening on the mechanical behavior is texture dependent and that incorporation of softening on basal systems only failed to replicate experimental observations in irradiated polycrystalline zircaloy. Analysis of a zircaloy texture representative of cladding material, which is oriented favorably for basal and prismatic slip, showed that the incorporation of basal softening only did not show the appropriate experimentally observed reduction in ductility. However, the introduction, in addition, of prismatic softening argued to result from irradiation effects, led to an accurate representation of the irradiated polycrystal stress-strain softening and failure response. It was also shown that strain hardening due to GND density development is small even local to a slip band and that macroscopic mechanical responses are dominated by irradiation induced softening. An optimized methodology for incorporating the basal and prismatic softening in crystal plasticity for irradiated zircaloy has been presented and validated against experimental observations. 
Representations of irradiated zircaloy in-service conditions were then investigated using strain-controlled and stress-controlled cyclic loading for three textured polycrystals. Under both strain and stress-controlled cyclic loading, irradiation softening led to the development of strain localization, and the formation of slip banding and its coalescence. In strain control, this was found to lead to localized ratcheting and macroscale softening, ultimately resulting in plastic shakedown. Stress-controlled cyclic loading, however, especially with non-zero mean applied stress, led to pronounced local and macroscale ratcheting, influenced profoundly by the irradiation softening.

A methodology has been presented for the assessment of structural integrity in irradiated zircaloy subject to cyclic loading which takes due account of crystallographic softening.

\section{Acknowledgements}

The authors thank Professor D. Rugg (Rolls-Royce) and Dr T.B. Britton for helpful discussions on irradiation effects in zircaloys.

\section{References}

1. Boyne, A., et al., Numerical simulation of irradiation hardening in Zirconium. Journal of Nuclear Materials, 2013. 438(1-3): p. 209-217.

2. Onimus, F. and J.L. Bechade, A polycrystalline modeling of the mechanical behavior of neutron irradiated zirconium alloys. Journal of Nuclear Materials, 2009. 384(2): p. 163-174.

3. Onimus, F., L. Dupuy, and F. Mompiou, In situ TEM observation of interactions between gliding dislocations and prismatic loops in Zr-ion irradiated zirconium alloys. Progress in Nuclear Energy, 2012. 57: p. 77-85.

4. Wei, X., J.R. Theaker, and M. Griffiths, Deformation Anisotropy of Annealed Zircaloy-2 as a Function of Fast Neutron Fluence. Zirconium in the Nuclear Industry: 15th International Symposium, 2009. 1505: p. 583-593.

5. Onimus, F., et al., A statistical TEM investigation of dislocation channeling mechanism in neutron irradiated zirconium alloys. Journal of Nuclear Materials, 2004. 328(2-3): p. 165179 .

6. Onimus, F., J.L. Bechade, and D. Gilbon, Experimental Analysis of Slip Systems Activation in Neutron-Irradiated Zirconium Alloys and Comparison with Polycrystalline Model Simulations. Metallurgical and Materials Transactions a-Physical Metallurgy and Materials Science, 2013. 44A: p. 45-60.

7. Lee, D., Adamson, R. B., Modelling of Localized Deformation in Neutron Irradiated Zircaloy-2. Zirconium in the Nuclear Industry, 1977. ASTM STP 633: p. 385-401.

8. Adamson, R.B., Bell, W. L., Effects of Neutron Irradiation and Oxygen Content on the Microstructure and Mechanical Properties of Zircaloy. Microstructure and Mechanical Behavior of Materials, Proceedings, 1986: p. 237-246.

9. Garde, A.M., Smith. G. P., Pirek, R. C., Effects of Hydride Precipitate Localization and Neutron Fluence on the Ductility of Irradiated Zircaloy-4. Zirconium in the Nuclear Industry: Eleventh International Symposium, 1996. ASTM STP 1295: p. 407-430.

10. Francillette, H., et al., Grain orientation effects in Zr702 alpha polycrystalline samples deformed in channel die compression at room temperature. Acta Materialia, 1998. 46(12): p. 4131-4142.

11. Wen, M.J., et al., Uniaxial ratcheting behavior of Zircaloy-4 tubes at room temperature. Materials \& Design, 2013. 46: p. 426-434.

12. Regnard, B., et al., Zirconium in the Nuclear Industry. ASTM STP 1423, 2002: p. 384.

13. Was, G.S., Fundamentals of radiation materials science : metals and alloys. 2007, Berlin: Springer. xxii, 827 p. 
14. Brown, L.M. and R.K. Ham, Strengthening Methods in Crystals. Applied Science Publishers Ltd., 1971: p. 594.

15. Drouet, J., et al., Dislocation dynamics simulations of interactions between gliding dislocations and radiation induced prismatic loops in zirconium. Journal of Nuclear Materials, 2014. 449(1-3): p. 252-262.

16. Ribis, J., et al., Experimental study and numerical modelling of the irradiation damage recovery in zirconium alloys. Journal of Nuclear Materials, 2010. 403(1-3): p. 135-146.

17. Onimus, F., et al., Plastic deformation of irradiated zirconium alloys: TEM investigations and micro-mechanical modeling. Zirconium in the Nuclear Industry: 14th International Symposium, 2005. 1467: p. 53-78.

18. Onchi, T., H. Kayano, and Y. Higashiguchi, Effect of Neutron-Irradiation on DeformationBehavior of Zirconium. Journal of Nuclear Science and Technology, 1977. 14(5): p. 359-369.

19. Lee, E.H., Elastic-Plastic Deformation at Finite Strains. Journal of Applied Mechanics, 1969. 36(1): p. 1-\&.

20. Peirce, D., R.J. Asaro, and A. Needleman, Material Rate Dependence and Localized Deformation in Crystalline Solids. Acta Metallurgica, 1983. 31(12): p. 1951-1976.

21. Huang, Y., A user-material subroutine incorporating single crystal plasticity in the abaqus finite element program. Harvard University Report, 1991. MECH 178.

22. Francillette, H., et al., Experimental an Predicted Texture Evolution in Zirconium Alloys Deformed in Channel Die Compression. Materials Science Forum, 1998. 273-275: p. 523528.

23. Erinosho, T.O. and F.P.E. Dunne, Lattice strain distributions due to elastic distortions and GND development in polycrystals. Journal of the Mechanics and Physics of Solids. 67(0): p. 62-86.

24. Arsenlis, A. and D.M. Parks, Crystallographic aspects of geometrically-necessary and statistically-stored dislocation density. Acta Materialia, 1999. 47(5): p. 1597-1611.

25. Coleman, C.E., D. Mills, and Vanderku.J, Deformation Parameters of Neutron-Irradiated Zircaloy-4 at 300 Degrees C. Canadian Metallurgical Quarterly, 1972. 11(1): p. 91-\&.

26. Xu, F., R.A. Holt, and M.R. Daymond, Modeling texture evolution during uni-axial deformation of Zircaloy-2. Journal of Nuclear Materials, 2009. 394(1): p. 9-19.

27. Gong, J.C., T.B. Britton, and A.J. Wilkinson, Single Crystal Microcantilever Testing. Private Communication (University of Oxford), 2014.

\section{Appendix A: Methodology to calibrate slip system strength}

Slip strengths determined in this study together with others reported in the literature are presented in Table 2, some of which have been determined from 'highly' textured polycrystal tests [22, 26], and others from single-crystal microcantilever test measurements [27]. The potential problem of the latter is that these measurements are potentially size-dependent, but in [24], size dependency has been removed. We observe that the strengths determined in this study for the basal and prismatic systems lie between those for the single crystal and 'highly textured' polycrystal results. The c+a value is higher but this is nonetheless consistent with the literature and of little significance in assessing softening in basal versus prismatic systems. In any case, the macro-level polycrystal uniaxial stress-strain response has been calculated using the CP model for the three independent slip strength sets shown in Fig. A1.

Table A1: Unirradiated $\mathrm{Zr}$ slip strengths and strength ratios in the literature

\begin{tabular}{|c||c||c|c||}
\hline Authors & $\tau^{\text {basal }}$ & $\tau^{\text {pris }}$ & $\tau^{\text {pyra }<c+a>}$ \\
\hline Xu et al. [26] & 155 & 100 & 320 \\
\hline \hline Gong et al. [27] & 180 & 147 & 452 \\
\hline
\end{tabular}




\begin{tabular}{|c|c||c||c||}
\hline Francillete et al. [22] & $\tau^{\text {basal }} / \tau_{\text {pris }}=1.3$ & $\tau^{\text {pris }} /{ }_{\tau_{\text {pris }}}=1$ & $\tau^{\text {pyra }<\mathrm{c}+\mathrm{a}>} /{ }_{\tau_{\text {pris }}}=4$ \\
\hline \hline This study & 162.5 & 125 & 500 \\
\hline
\end{tabular}

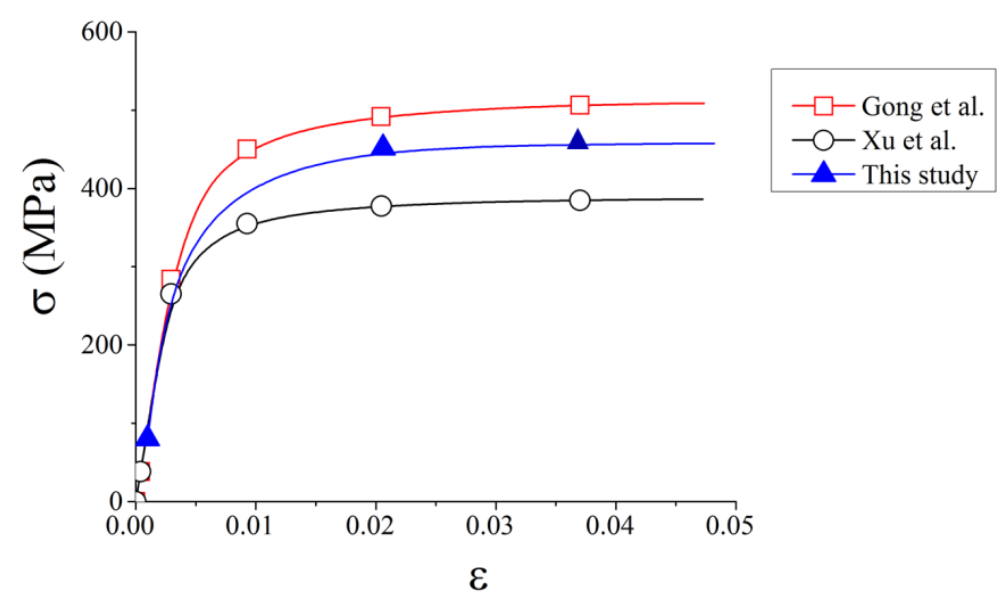

Fig. A 1: Unirradiated macroscopic stress strain response for the range of initial CRSS in the literature

\section{Appendix B: Example showing the effect of planar boundary conditions on simulations}

In the polycrystal calculations, the negative planes are restrained to be planar in order to impose (uniaxial) constraint on the deformation, and the positive planes are unconstrained in order to be representative of free surfaces. This is unlikely to be key in determining the role and necessity of prismatic softening in $\mathrm{Zr}$ softening in addition to basal softening. In order to demonstrate this, a polycrystal analysis (Texture $\mathrm{C}$ under uniaxial straining) has been performed in which all planes are constrained to remain planar and the resulting slip field is presented in Fig. B1.

(a)

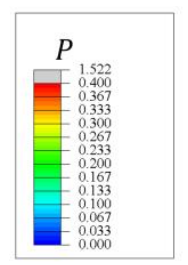

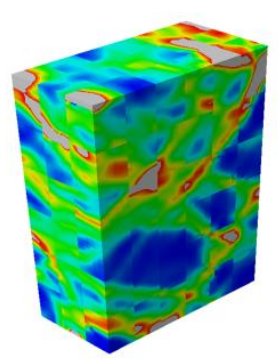
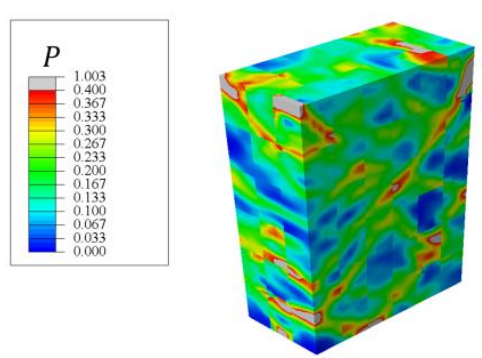

Fig. B 1: Slip field (mid-section shown) for irradiated Texture $C$ under uniaxial straining at 5 percent strain showing the example in which all positive surfaces are left unconstrained (a) and the example in which all planes are constrained to remain planar (b)

Fig. B1a shows the slip field for the example in which the positive planes are left unconstrained while Fig. B1b shows the case in which all planes are constrained to remain planar. It is clear from Fig. B1 that the boundary conditions chosen (i.e. constraining all faces 
to remain planar or otherwise) do not significantly affect the overall slip response, nor the slip localisation. Some small differences in spatial distribution are apparent, but the localization and softening processes are driven from within the bulk of the polycrystal and the boundary conditions chosen on the surface do not significantly affect the rates of reduction in ductility resulting from incorporating softening on differing slip systems.

\section{Appendix C: Validation of mesh refinement used in the polycrystal model}

The polycrystal model shown in Fig. 9a consists of 216 grains with each grain having $5 \times 5 \times 10$ elements. This level of refinement was chosen as sufficient to capture the mechanical localization response under uniaxial straining as shown in Fig. C1. The polycrystal was subjected to uniaxial straining in the Y-direction shown in Fig. 9a and the mechanical response for three levels of mesh refinement is shown in Fig. C1. It is evident that only small differences in stress magnitude are seen by choosing higher levels of refinement and the yield point and onset of localization marked by a drop in the stress-strain response are sufficiently captured. Hence, $5 \times 5 \times 10$ elements per grain are chosen as a suitable refinement for all polycrystal simulations for reasons of computational efficiency.

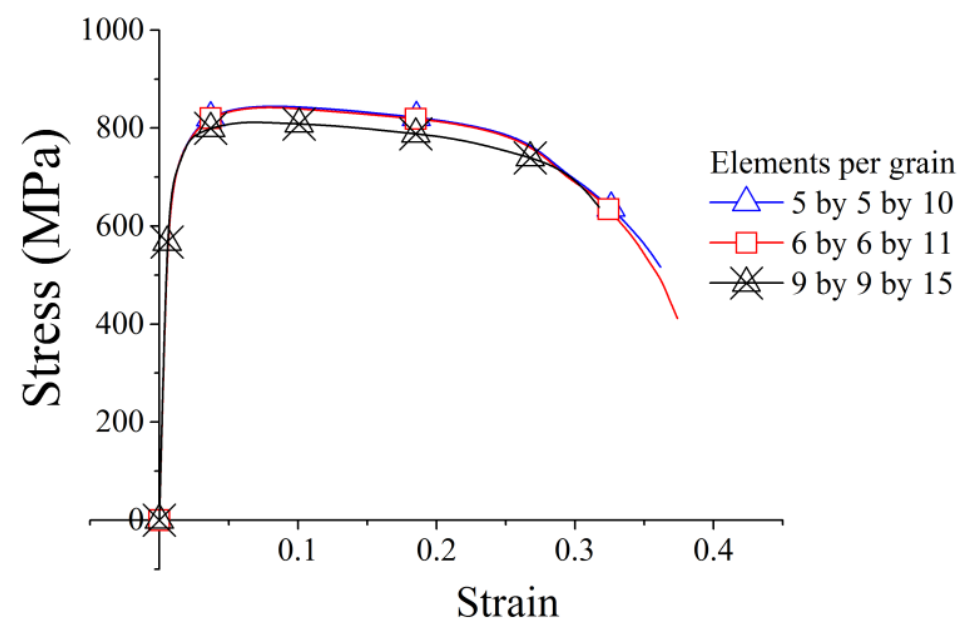

Fig. C 1: Validation of the mesh refinement used in the polycrystal simulations. Three levels of refinement shown are subjected to uniaxial straining with only small differences observed with increasing refinement.

\section{Appendix D: Attempt to calibrate the damage model using basal softening only}

In the example considered, extreme cases of softening are prescribed on the basal system such that at the critical value of initiating softening, the strength on the basal system is immediately reduced from the irradiated $(271.5 \mathrm{MPa})$ to the unirradiated $(162.5 \mathrm{MPa})$ value. That is:

$$
\gamma^{\text {basal }}=\left\{\begin{array}{ll}
271.5 \mathrm{MPa} & \gamma^{\text {basal }} \leq \gamma^{\text {crit }} \\
162.5 \mathrm{MPa} & \gamma^{\text {basal }}>\gamma^{\text {crit }}
\end{array} \text { and } \gamma^{\text {crit }}=0,0.01 \text { and } 0.03 .\right.
$$


It is clear from Fig. D1 that whilst accounting for basal softening only may approximately capture the yield strength of the irradiated experimental response, it cannot match the reduced ductility and for this reason, it is hypothesized that other systems potentially contribute to the lower ductility in the irradiated polycrystal.

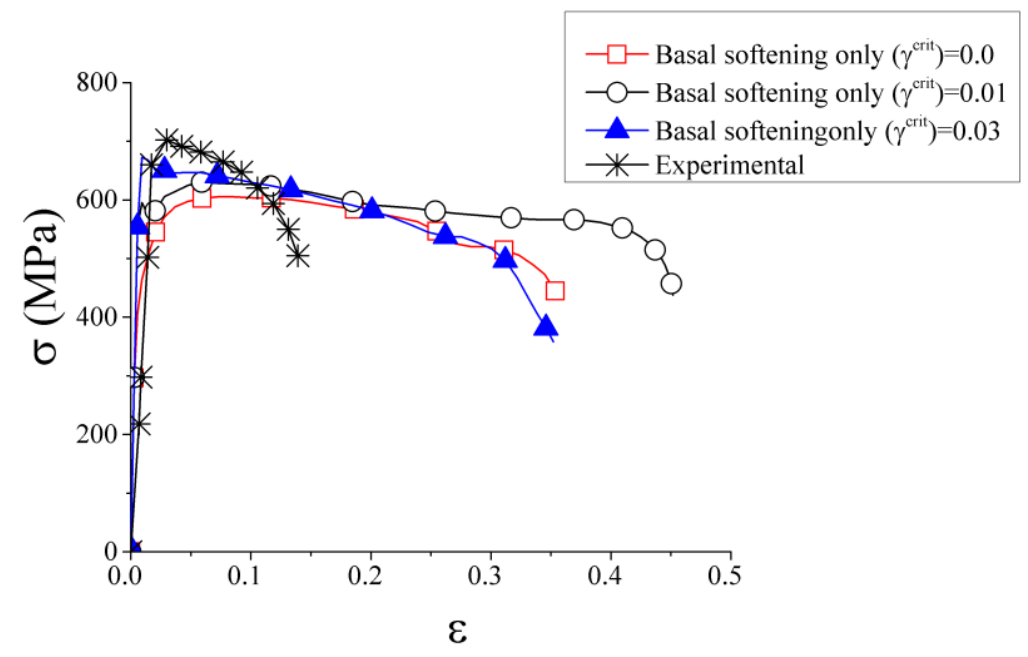

Fig. D 1: Attempt to calibrate the damage model using basal softening only.

Therefore, it can be inferred from Figure D1 that other slip systems (prismatic and perhaps even pyramidals) are important to capturing irradiation effects since the reduction in ductility could not be captured even by adopting extreme rates of softening on basal systems only. 